\title{
Combining Lift-and-Project and Reduce-and-Split
}

\author{
Egon Balas* \\ Tepper School of Business, Carnegie Mellon University, PA \\ Email: eb17@andrew.cmu.edu \\ Gérard Cornuéjols ${ }^{\dagger}$ \\ Tepper School of Business, Carnegie Mellon University, PA \\ Email: gc0v@andrew.cmu.edu \\ Tamás Kis ${ }^{\ddagger}$ \\ Computer and Automation Research Institute, \\ Hungarian Academy of Sciences, Hungary \\ Email: tamas.kis@sztaki.hu \\ Giacomo Nannicini ${ }^{\S}$ \\ Tepper School of Business, Carnegie Mellon University, PA \\ Email: nannicin@andrew.cmu.edu
}

January 24, 2012

\begin{abstract}
Split cuts constitute a class of cutting planes that has been successfully employed by the majority of Branch-and-Cut solvers for Mixed Integer Linear Programs. Given a basis of the LP relaxation and a split disjunction, the corresponding split cut can be computed with a closed form expression. In this paper, we use the Lift-and-Project framework [11] to provide the basis, and the Reduce-andSplit algorithm [19] to compute the split disjunction. We propose a cut generation algorithm that starts from a Gomory Mixed Integer cut and alternates between Lift-and-Project and Reduce-and-Split in order to strengthen it. This paper has two main contributions. First, we extend the Balas and Perregaard procedure for strengthening cuts arising from split disjunctions involving one variable, to split disjunctions on multiple variables. Second, we apply the Reduce-and-Split algorithm to non-optimal bases of the LP relaxation. We provide detailed computational testing of the proposed methods.

Keywords: Integer Programming, Computational Analysis, Branch-and-Cut, Liftand-Project.
\end{abstract}

\footnotetext{
*Supported by NSF grant CMMI1024554 and ONR grant N00014-09-1-0033.

${ }^{\dagger}$ Supported by NSF grant CMMI1024554 and ONR grant N00014-09-1-0033.

¥Supported by the Hungarian Research Fund OTKA K76810.

$\S$ Supported by an IBM Fellowship.
} 


\section{Introduction}

Mixed Integer Linear Programs (MILPs), i.e. mathematical programs with linear objective and constraints and both continuous and integer variables, arise in a number of real-world applications, and their solution is therefore of great practical interest. The most successful softwares for solving general MILPs utilize a Branch-and-Cut algorithm, which combines cutting planes and Branch-and-Bound. Several classes of cutting planes used by these softwares, such as Gomory Mixed Integer (GMI) cuts [20], Mixed Integer Rounding (MIR) cuts [23] and Lift-and-Project cuts [8], fall into the category of split cuts [18], that is, disjunctive cuts derived from two parallel hyperplanes. It was shown in [4] that every split cut can be generated as an intersection cut [5] from an appropriate choice of a basis of the LP relaxation and a split disjunction. The advantage of generating split cuts as intersection cuts is that we can use closed form expressions, without having to resort to disjunctive programming [6]. In this paper, we propose a split cut generation procedure that is based on Lift-and-Project $[9,11]$ and Reduce-and-Split $[3,19]$. In particular, we use the former to select a basis of the LP relaxation, and the latter to compute a split disjunction.

Lift-and-Project (L\&P) cuts have been successfully used in the Branch-and-Cut framework since the 90s [9]. A significant improvement in their practical performance came a few years later, when a procedure to generate L\&P cuts without solving the higherdimensional Cut Generating Linear Program (CGLP) was introduced by Balas and Perregaard [11]. This procedure starts with a split cut arising from a violated two-term disjunction involving a single variable and the optimal basis of the LP relaxation (in other words, a GMI cut), and mimicks the solution of the CGLP by performing pivots in the original simplex tableau. The procedure yields a new (possibly infeasible) basis, from which a stronger cut than the initial GMI cut can be generated. This procedure has been incorporated into commercial solvers like Xpress-MP [24], MOPS [25], and several versions of it have been implemented in the open source project COIN-OR Cgl [16]. One of the main contributions of this paper consists in an extension of this procedure to split cuts arising from general split disjunctions, i.e. any violated two-term disjunction involving an integral linear combination of integer variables. This yields a procedure that, given any split disjunction and any basis, produces a different basis that gives rise to a stronger cut.

In order to apply this extended L\&P procedure, we need a method for generating an initial split disjunction. We use the Reduce-and-Split (R\&S) algorithm for this purpose. $\mathrm{R} \& \mathrm{~S}$, first introduced in [3] and then revisited in [19], is a cut generation algorithm that starts from an optimal LP basis and a split disjunction on one variable, and computes a split disjunction involving several variables that (heuristically) yields a better cut. Therefore, we have an algorithm to produce split disjunctions, which can be used to initialize the L\&P procedure.

Another contribution of this paper is that we apply R\&S on non-optimal, possibly infeasible, tableaux. As a consequence, we have a procedure that, given any split disjunction and any basis, produces a new, often better split disjunction for cut generation. Thus, we can alternate between the two procedures introduced in this paper, and iteratively change both the basis and the split disjunction from which a split cut is generated.

We perform extensive computational experiments on a set of benchmark MILPs to assess the effectiveness of our ideas. Our computational results show that, within a Cut-and-Branch framework, the combination of the two cut generation algorithms yields stronger cutting planes than L\&P or R\&S alone. We obtain the best results by alternating between the two more than once. 
The rest of this paper is organized as follows. In Section 2 we introduce our notation and provide the necessary theoretical background. In Section 3 we review in more detail the Lift-and-Project procedure introduced in [11], and extend it to general split disjunctions. Section 4 reviews the Reduce-and-Split method, and discusses its application on non-optimal bases of the LP relaxation. In Section 5 we describe our cut generation algorithm, which alternates between the Lift-and-Project and the Reduce-and-Split procedures. Section 6 presents an extensive computational evaluation. Section 7 concludes the paper. Detailed tables of results can be found in the Appendix.

\section{Notation and preliminaries}

We are considering a MILP of the form:

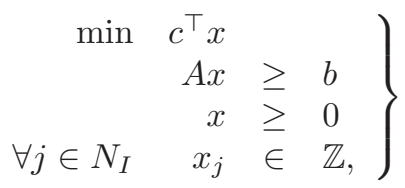

where $A \in \mathbb{R}^{m \times n}, b \in \mathbb{R}^{m}, c \in \mathbb{R}^{n}, N:=\{1, \ldots, n\}, N_{I}:=\{1, \ldots, p\}$ with $p \leq n$, and where upper bounding constraints are subsumed by $A x \geq b$. In the sequel (LP) will stand for the linear programming relaxation of (MILP). A split cut for (MILP) is a valid inequality derived from a disjunction of the form

$$
\pi x \leq \pi_{0} \quad \vee \quad \pi x \geq \pi_{0}+1,
$$

where $\pi_{j}$ is integer for $j \in N_{I}, \pi_{j}=0$ for $j \in N \backslash N_{I}$, and $\pi_{0}$ is an integer whose value depends on the fractional point we want to cut off. For a given fractional point $\bar{x}, \pi_{0}$ is chosen so as to have

$$
\pi_{0}<\pi \bar{x}<\pi_{0}+1,
$$

which yields $\pi_{0}=\lfloor\pi \bar{x}\rfloor$. If $\bar{x}$ is a basic solution to (LP) such that $\bar{x}_{k}$ is fractional and $k \in N_{I}$, then

$$
x_{k} \leq\left\lfloor\bar{x}_{k}\right\rfloor \vee x_{k} \geq\left\lfloor\bar{x}_{k}\right\rfloor+1
$$

is an elementary disjunction and it is a special case of (1).

A GMI cut from (1) (or from (3)) can be derived as follows. Rewrite (LP) in standard form:

$$
\left.\min \begin{array}{rl}
c^{\top} x & \\
(A,-I) x & =b \\
x & \geq 0
\end{array}\right\}
$$

where $x \in \mathbb{R}^{n+m}$ and the last $m$ components are surplus variables. Let $\bar{x}$ be a basic solution, $B(\bar{x})$ the set of indices of basic variables, and $J(\bar{x})=N \backslash B(\bar{x})$ the set of nonbasic variables. Then, the corresponding simplex tableau can be written as:

$$
x_{i}=\bar{x}_{i}-\sum_{j \in J(\bar{x})} \bar{a}_{i j} x_{j} \quad \forall i \in B(\bar{x}) .
$$

Let $B_{I}(\bar{x})=B(\bar{x}) \cap N_{I}, J_{I}(\bar{x})=J(\bar{x}) \cap N_{I}, J_{C}(\bar{x})=J(\bar{x}) \backslash N_{I}$ be the sets of integer basic variables, integer nonbasic variables and continuous nonbasic variables, respectively. Consider a linear combination with integer coefficients $\pi_{i}$ of those rows of (4) where 
$i \in B_{I}(\bar{x}):$

$$
\sum_{i \in B_{I}(\bar{x})} \pi_{i} x_{i}=\hat{x}-\sum_{j \in J(\bar{x})} \hat{a}_{j} x_{j}
$$

where

$$
\begin{aligned}
\hat{x} & =\sum_{i \in B_{I}(\bar{x})} \pi_{i} \bar{x}_{i} \\
\hat{a}_{j} & =\sum_{i \in B_{I}(\bar{x})} \pi_{i} \bar{a}_{i j} \text { for } j \in J(\bar{x}) .
\end{aligned}
$$

Let $\pi_{0}=\lfloor\hat{x}\rfloor$, and define $f_{0}=\hat{x}-\pi_{0}, f_{j}=\hat{a}_{j}-\left\lfloor\hat{a}_{j}\right\rfloor$ for all $j \in J$. If $\hat{x} \notin \mathbb{Z}$, we can derive from (5) the following valid inequality for (MILP):

$$
\begin{gathered}
\sum_{j \in J_{I}(\bar{x}): f_{j} \leq f_{0}} \frac{f_{j}}{f_{0}} x_{j}+\sum_{j \in J_{I}(\bar{x}): f_{j}>f_{0}} \frac{1-f_{j}}{1-f_{0}} x_{j}+ \\
\sum_{j \in J_{C}(\bar{x}): \hat{a}_{j} \geq 0} \frac{\hat{a}_{j}}{f_{0}} x_{j}-\sum_{j \in J_{C}(\bar{x}): \hat{a}_{j}<0} \frac{\hat{a}_{j}}{1-f_{0}} x_{j} \geq 1 .
\end{gathered}
$$

This inequality is the GMI cut associated with the equation obtained through the row multipliers $\pi_{i}$; its validity is shown in [20]. Choosing $\pi_{k}=1, \pi_{i}=0 \forall i \neq k$ yields the GMI cut from row $k$ of (4).

The derivation of (7) from (5) proceeds as follows. Consider the disjunction obtained by substituting the right hand side of (5) into (1):

$$
\hat{x}-\sum_{j \in J(\bar{x})} \hat{a}_{j} x_{j} \leq \pi_{0} \quad \vee \quad \hat{x}-\sum_{j \in J(\bar{x})} \hat{a}_{j} x_{j} \geq \pi_{0}+1 .
$$

Rewriting this gives

$$
\sum_{j \in J(\bar{x})} \hat{a}_{j} x_{j} \geq f_{0} \quad \vee \sum_{j \in J(\bar{x})}\left(-\hat{a}_{j}\right) x_{j} \geq 1-f_{0} .
$$

The disjunctive cut obtained from the latter disjunction is

$$
\sum_{j \in J(\bar{x}): \hat{a}_{j} \geq 0} \frac{\hat{a}_{j}}{f_{0}} x_{j}-\sum_{j \in J(\bar{x}): \hat{a}_{j}<0} \frac{\hat{a}_{j}}{1-f_{0}} x_{j} \geq 1 .
$$

By applying the integer modularization procedure of Balas [6] and Balas and Jeroslow [7] to (8), we obtain (7). Alternatively, (7) is the disjunctive cut from the following general disjunction, obtained from $\pi$ by strengthening the coefficients on the nonbasic integer variables:

$$
\hat{\pi}_{i}= \begin{cases}\pi_{i} & \text { if } i \in B_{I}(\bar{x}) \\ \left\lfloor\hat{a}_{i}\right\rfloor & \text { if } i \in J_{I}(\bar{x}) \text { and } f_{i} \leq f_{0} \\ \left\lceil\hat{a}_{i}\right\rceil & \text { if } i \in J_{I}(\bar{x}) \text { and } f_{i}>f_{0} \\ 0 & \text { otherwise. }\end{cases}
$$

\section{Lift-and-Project on general disjunctions}

We start this section by reviewing the original Lift-and-Project procedure, then we introduce one of the main contributions of this paper, namely the extension of the Lift- 
and-Project procedure on the original simplex tableau to general two-term disjunctions.

\subsection{Review of Lift-and-Project}

Lift-and-Project cuts were introduced in [8], and [9]. These are cuts obtained from a two-term disjunction of the form

$$
\left(\begin{array}{rl}
A x & \geq b \\
x & \geq 0 \\
-x_{k} & \geq 0
\end{array}\right) \vee\left(\begin{array}{rl}
A x & \geq b \\
x & \geq 0 \\
x_{k} & \geq 1
\end{array}\right)
$$

where $1 \leq k \leq p$ with $0<\bar{x}_{k}<1$. A lift-and-project cut from this disjunction is derived by solving the so-called cut generating linear program (CGLP) $)_{k}$ :

$$
\begin{aligned}
& \min \alpha \bar{x}-\beta
\end{aligned}
$$

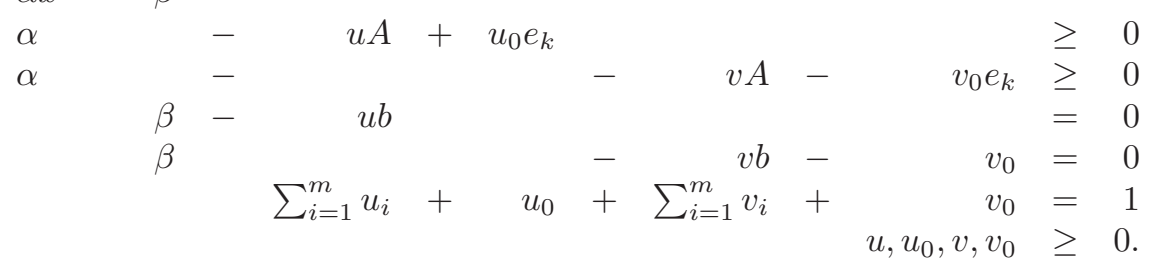

Here, $e_{k}$ is the $k$-th unit vector. The objective function maximizes the violation of the cut $\alpha x \geq \beta$ in point $\bar{x}$. The last equation is a normalization constraint which ensures that $(\mathrm{CGLP})_{k}$ always has a finite optimum.

Balas and Perregard observed that (CGLP) $)_{k}$ can be solved to optimality in the original simplex tableau [11]. In fact, they describe the precise correspondence between the feasible bases of (CGLP) $)_{k}$ and the bases of $(\mathrm{LP})_{s}$. Notice that in this correspondence, the bases of $(\mathrm{LP})_{s}$ are generally infeasible, i.e., in the corresponding LP solution, $x$ may have negative coordinates.

The L\&P procedure is illustrated in Fig. 1, where the basic solution $\bar{x}$ is to be cut off, but the corresponding cut $\alpha x \geq \alpha_{0}$ is weaker than the one that can be derived after pivoting to $\bar{x}^{\prime}$ and deriving the cut $\alpha^{\prime} x \geq \alpha_{0}^{\prime}$ from that (infeasible) basic solution. The rays $r^{i}$ for $i=1,2$ correspond to the non-basic columns of the simplex tableau in the basic solution $\bar{x}$, whereas $r^{1}$ and $r^{3}$ are those in the basis corresponding to $\bar{x}^{\prime}$.

\subsection{Lift-and-Project applied to general split disjunctions}

For the sake of compact notation, we define $(\tilde{A}, \tilde{b})$ as

$$
(\tilde{A}, \tilde{b}):=\left(\begin{array}{cc}
A & b \\
I & 0
\end{array}\right)
$$

where $I$ is the $n \times n$ identity matrix. In order to apply the Lift-and-Project procedure to a disjunction of the form (1), one could simply formulate the (CGLP) corresponding to

$$
\left(\begin{array}{ccc}
\tilde{A} x & \geq & \tilde{b} \\
-\pi x & \geq & -\pi_{0}
\end{array}\right) \bigvee\left(\begin{array}{ccc}
\tilde{A} x & \geq & \tilde{b} \\
\pi x & \geq & \pi_{0}+1
\end{array}\right) .
$$

However, in order to take full advantage of the correspondence between the (CGLP) and the (LP) established in [11], it will be preferable to introduce a new integer variable 


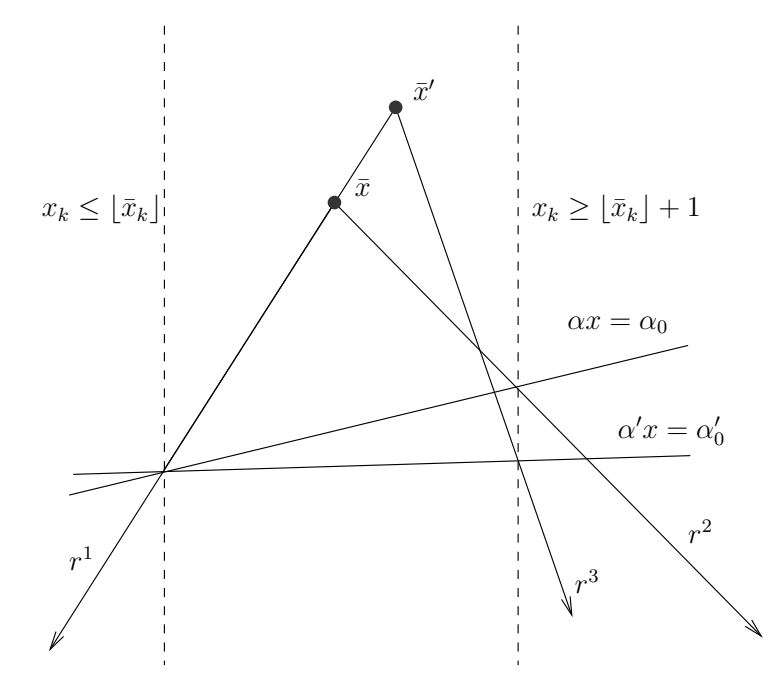

Figure 1: Illustration of the Lift-and-Project procedure.

$x_{n+m+1}$ to represent the difference between $\pi x$ and $\pi_{0}$ :

$$
\pi x-x_{n+m+1}=\pi_{0}
$$

Notice that, since $\pi x$ is integer in any feasible solution of (MILP) and $\pi_{0}$ is integer, $x_{n+m+1}$ has to be integer as well. Moreover, if $x_{n+m+1} \leq 0$, then $\pi x \leq \pi_{0}$, while if $x_{n+m+1} \geq 1$, then $\pi x \geq \pi_{0}+1$, as desired. Using the new variable, we can rewrite (10):

$$
\left(\begin{array}{rlc}
\tilde{A} x & \geq & \tilde{b} \\
\pi x-x_{n+m+1} & = & \pi_{0} \\
x_{n+m+1} & \leq 0
\end{array}\right) \bigvee\left(\begin{array}{rl}
\tilde{A} x & \geq \tilde{b} \\
\pi x-x_{n+m+1} & =\pi_{0} \\
x_{n+m+1} & \geq 1
\end{array}\right)
$$

The important difference from the previous applications of the Lift-and-Project procedure to single rows (4) of the simplex tableau is the following. The equation (11) is constructed in order to derive a cut from it. Once the cut is derived, the equation is no longer needed and therefore it is discarded, along with the variable $x_{n+m+1}$. On the other hand, the variable $x_{n+m+1}$, and its expression in terms of the current nonbasic variables, is needed throughout the pivoting process carried out in order to (implicitly) optimize the CGLP. Thus, we have to add a new row to the optimal (LP) tableau and keep it until the cut is optimized. This could be done by simply adding the equation $\pi x-x_{n+m+1}=\pi_{0}$ to the constraint set of (MILP), and then computing the amended simplex tableau corresponding to the current basis. Instead, one can derive the new row as a closed form expression.

\subsection{Proposition}

Let $\left(A_{B}, A_{J}\right)$ be the partition of $(A,-I)$ into basic and nonbasic columns. Then the expression for $x_{n+m+1}=\pi x-\pi_{0}$ in terms of the nonbasic variables is

$$
x_{n+m+1}+\left(\pi_{B} A_{B}^{-1} A_{J}-\pi_{J}\right) x_{J}=\left(\pi_{B} A_{B}^{-1}\right) b-\pi_{0}
$$


Proof. The simplex tableau corresponding to the basis indexed by $B$ is

$$
x_{B}+A_{B}^{-1} A_{J} x_{J}=A_{B}^{-1} b .
$$

If $\pi=\left(\pi_{B}, \pi_{J}\right)$ and $\pi_{j}=0$ for all $j \in N \backslash N_{I}$, then $\pi x-x_{n+m+1}=\pi_{0}$ can be written as

$$
-x_{n+m+1}+\pi_{B} x_{B}+\pi_{J} x_{J}=\pi_{0} .
$$

Appending this equation to $\left(A_{B}, A_{J}\right) x=b$ gives

$$
\begin{aligned}
& A_{B} x_{B}+A_{J} x_{J}=b \\
& \pi_{B} x_{B}-x_{n+m+1}+\pi_{J} x_{J}=\pi_{0} .
\end{aligned}
$$

The inverse of the $(m+1) \times(m+1)$ matrix $\left(\begin{array}{cc}A_{B} & 0 \\ \pi_{B} & -1\end{array}\right)$ is $\left(\begin{array}{cc}A_{B}^{-1} & 0 \\ \pi_{B} A_{B}^{-1} & -1\end{array}\right)$. Multiplying (14) with this augmented basis inverse gives

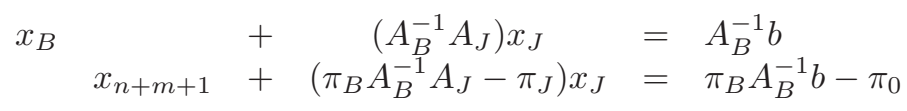

The new source row (13) could of course be used to directly generate a generalized GMI cut; instead, we apply to it the L\&P procedure of [11], or one of its variants discussed in $[12]$ in order to obtain a stronger cut. Such a cut will be valid throughout the search tree in case of a mixed 0-1 program, but only at the descendants of the current search tree node for a general mixed integer program (see [10]).

\subsection{Implementation of the generalized Lift-and-project proce- dure}

It is not too difficult to modify any implementation of the L\&P cut generation procedure that works on the simplex tableau and strengthens cuts derived from a disjunction (3), so that it can strengthen split cuts derived from a more general split disjunction (1). Namely, the L\&P procedure must have a subroutine to extract the source row from the simplex tableau before pivoting and after each pivot. This is usually done by using the basis inverse, which is typically readily available: most Branch-and-Cut (or Cut-and-Branch) solvers use the revised dual simplex method, which maintains the basis inverse rather than the full simplex tableau. It suffices to modify this subroutine so that it computes the source row using (13). This can be implemented rather efficiently using the standard Ftran or Btran subroutines, available in many commercial and free state-of-the-art LP solvers.

\section{Reduce-and-Split from non-optimal bases}

As in Section 3, we first recall the basic concepts of Reduce-and-Split, then we discuss our contribution: the application of Reduce-and-Split on non-optimal bases. 


\subsection{Review of Reduce-and-Split}

The idea of looking for a linear combination (5) of rows of the simplex tableau (4) to generate strong cutting planes is not new in the integer programming literature: see e.g. $[3,14,19]$. As discussed in Section 2, every equation (5) such that $\sum_{i \in B_{I}(\bar{x})} \pi_{i} \bar{x}_{i}$ is fractional yields a valid GMI cut. Here, $\bar{x}$ need not be an optimal solution to (LP); however, this is the only case that is typically studied in the literature. In the next section we consider the case where $\bar{x}$ is basic but not optimal for (LP). In particular, our discussion focuses on the case where $\bar{x}$ is a basic solution for a L\&P tableau, i.e. a (possibly primal infeasible) tableau obtained by pivoting following the L\&P procedure.

We now review the R\&S algorithm, as given in [19]. Let $\bar{x}$ be the optimal solution to (LP). R\&S first determines a working set of continuous nonbasic columns $J_{W} \subset$ $J_{C}(\bar{x})$, then generates an integral combination (5) of the rows of the simplex tableau corresponding to the basic variables in $B_{I}(\bar{x})$ by minimizing:

$$
\min _{\pi \in \mathbb{Z}^{\left|B_{I}(\bar{x})\right|}}\left\|\left(\hat{a}_{j}\right)_{j \in J_{W}}\right\|_{2}
$$

where $\hat{a}_{j}$ is defined as in (6). Observe that the linear combination (5) can involve rows with an integer valued basic variable, as long as $\sum_{i \in B_{I}(\bar{x})} \pi_{i} \bar{x}_{i}$ is fractional. The minimization problem (15) yields row multipliers $\pi_{i}$ from which we derive (7). As can be seen from (7), small $\hat{a}_{j}$ on continuous nonbasic columns should yield good (i.e. small) cut coefficients on the corresponding variables. Note that our aim is to improve the cut coefficients on continuous variables only. The reason for focusing on continuous variables only is that the cut coefficients on integer variables are much more difficult to control, because of the modular arithmetic involved in their expression (see (7)). (15) is solved by relaxing integrality on $\pi$, determining the optimal continuous multipliers (imposing an additional normalization constraint to avoid the all zero solution), then rounding the fractional components $\pi$ to the nearest integer. In [19] it is experimentally shown that variables with small reduced cost are good candidates for the set $J_{W}$, as they yield cuts which close a larger integrality gap in practice. Furthermore, instead of considering all rows whose corresponding basic variable is in $B_{I}(\bar{x})$, it is shown that better results can be obtained by considering only a subset of carefully chosen rows. Since we are interested in finding a linear combination that yields small coefficients, the chosen rows should ideally be linearly dependent or almost.

In its default configuration, the Reduce-and-Split cut generation algorithm proceeds as follows: for each row $r$ of the simplex tableau with an integer basic variable $x_{k}$, a subset of columns $J_{W} \subset J_{C}(\bar{x})$ and a subset of rows other than $r$ with a basic integer variable is chosen. Then, a linear combination of these rows is sought using the procedure outlined above. The normalization condition to avoid the all zero solution to (15) consists in requiring $\pi_{k}=1$. This loop is iterated several times using different strategies to select $J_{W}$ and the set of rows. This is the basic variant of the Reduce-and-Split algorithm: we refer to [19] for a thorough discussion.

The geometric interpretation is as follows. R\&S keeps the basis $B(\bar{x})$ fixed, and tries to modify the split disjunction (1) in order to obtain a cut with stronger coefficients. This is exemplified in Figure 2: the elementary disjunction $x_{k} \leq\left\lfloor\bar{x}_{k}\right\rfloor \vee x_{k} \geq\left\lfloor\bar{x}_{k}\right\rfloor+1$, which yields the cut $\alpha x \geq \alpha_{0}$, is modified to obtain a stronger cut $\alpha^{\prime} x \geq \alpha_{0}^{\prime}$ (from disjunction $\left.\pi x \leq \pi_{0} \vee \pi x \geq \pi_{0}+1\right)$. Again, the rays $r^{1}$ and $r^{2}$ correspond to the non-basic columns of the simplex tableau with basic solution $\bar{x}$. In terms of (9), R\&S acts on the coefficients $\pi_{i}$ of the disjunction on the basic integer variables. 


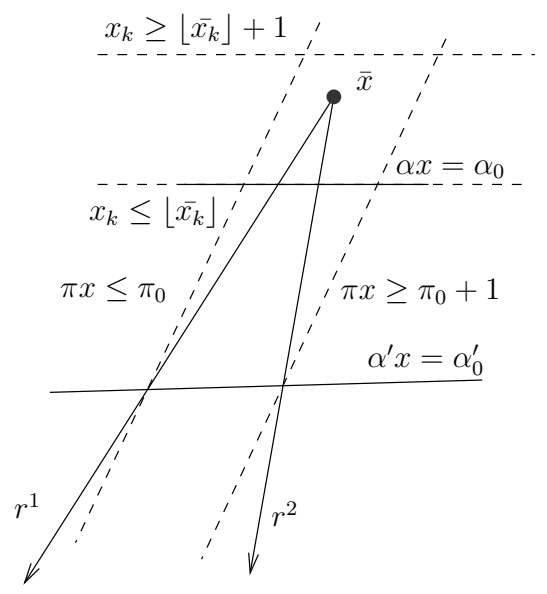

Figure 2: Illustration of the Reduce-and-Split procedure.

\subsection{Modifications to Reduce-and-Split}

In Section 3 we proposed a method to start with any split disjunction, and modify the basis via L\&P to obtain a stronger cut. What we want to do now is to use the basis computed by L\&P, and modify ("tilt") the split disjunction to derive a better cut.

A problem arises: a cut derived from a non-optimal basis of (LP) will certainly be valid, but how do we make sure that it will be violated by the point that we want to cut off? To show why such a cut might not be violated, we need to introduce some notation. Let $\bar{x}$ be the optimal solution to (LP), where the corresponding optimal tableau $\bar{A}$ has elements $\bar{a}_{i j}$. Let $\bar{x}^{\prime}$ be the basic solution associated with the tableau $\bar{A}^{\prime}$ (with elements $\bar{a}_{i j}^{\prime}$ ) obtained by applying L\&P starting from $\bar{x}$. A GMI obtained from tableau $\bar{A}$ has the form

$$
\sum_{j \in J(\bar{x})} \alpha_{j} x_{j} \geq \alpha_{0},
$$

with $\alpha_{j} \geq 0, \alpha_{0}>0$. Since $\bar{x}_{j}=0 \forall j \in J(\bar{x})$, this cut is violated by $\bar{x}$. On the other hand, a split cut obtained as a GMI cut from tableau $\bar{A}^{\prime}$ has the form:

$$
\sum_{j \in J\left(\bar{x}^{\prime}\right)} \alpha_{j}^{\prime} x_{j} \geq \alpha_{0}^{\prime},
$$

and cuts off $\bar{x}^{\prime}$ but is not necessarily violated by $\bar{x}$. Indeed, $\bar{x}_{j}=0 \forall j \in J\left(\bar{x}^{\prime}\right) \cap J(\bar{x})$ but $\bar{x}_{j} \geq 0 \forall j \in J\left(\bar{x}^{\prime}\right) \cap B(\bar{x})$, therefore the left hand side may be $>0$ at $\bar{x}$. The cut will be violated if and only if $\sum_{j \in J\left(\bar{x}^{\prime}\right) \cap B(\bar{x})} \alpha_{j}^{\prime} \bar{x}_{j}<\alpha_{0}^{\prime}$. This suggests that we should aim for small (hopefully zero) cut coefficients on the columns with indices in $J\left(\bar{x}^{\prime}\right) \cap B(\bar{x})$. In Figure 3, we picture an example of a non violated cut: the L\&P cut obtained from the new basic solution $\bar{x}^{\prime}$ and the initial disjunction $x_{k} \leq\left\lfloor\bar{x}_{k}\right\rfloor \vee x_{k} \geq\left\lfloor\bar{x}_{k}\right\rfloor+1$ cuts off $\bar{x}$ by construction, but as soon as the disjunction is modified, we are only guaranteed to cut off $\bar{x}^{\prime}$ (as shown by the cut $\alpha^{\prime} x \geq \alpha_{0}^{\prime}$ ).

In order to generate cuts from $\bar{A}^{\prime}$ that are likely to cut off $\bar{x}$, we modify the R\&S algorithm as follows. Let $B^{*}=J\left(\bar{x}^{\prime}\right) \cap B(\bar{x})$ be the set of variables which are basic in the optimal LP tableau but are nonbasic in the L\&P tableau on which we apply 


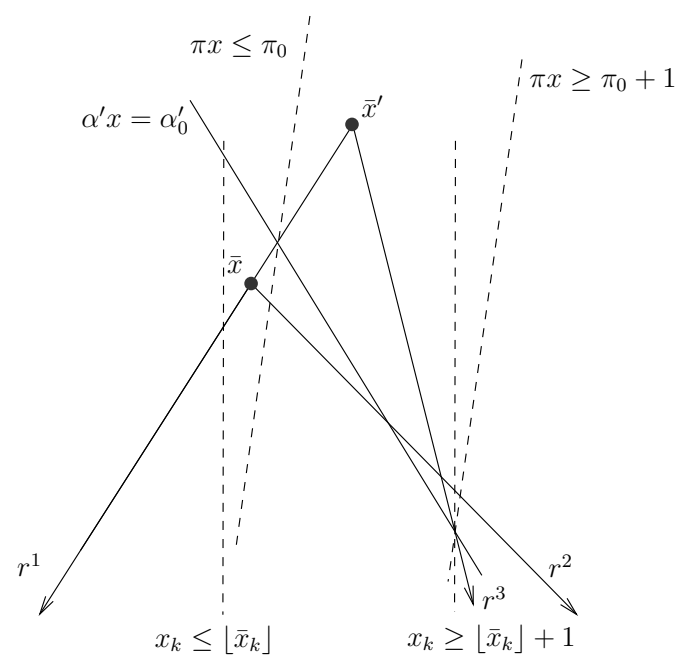

Figure 3: A Reduce-and-Split cut from the disjunction $\pi x \leq \pi_{0} \vee \pi x \geq \pi_{0}+1$, obtained from the tableau associated with the basic solution $\bar{x}^{\prime}$, that does not cut off the point $\bar{x}$.

R\&S. Given $J_{W} \subset J_{C}\left(\bar{x}^{\prime}\right)$ (e.g. using one of the techniques described in [19]) and scalars $\sigma_{j}>0 \forall j \in J_{W} \cup B^{*}$, we compute:

$$
\min _{\pi \in \mathbb{Z}^{\left|B_{I}\left(\bar{x}^{\prime}\right)\right|}}\left\|\sum_{i \in B_{I}\left(\bar{x}^{\prime}\right)} \pi_{i} d_{i}^{\prime}\right\|_{2},
$$

where $d_{i}^{\prime}=\left(\sigma_{j} \bar{a}_{i j}^{\prime}\right)_{j \in J_{W} \cup B^{*}}$; in other words, $d_{i}^{\prime}$ are rows of a submatrix of $\bar{A}^{\prime}$ (corresponding to the set of columns $J_{W} \cup B^{*}$ ), where each column is rescaled with multipliers $\sigma_{j}$. The effect of these multipliers is to modify the importance of the columns when determining $\pi$ that minimizes the norm in (16), by increasing it (if $\sigma_{j}$ is large) or decreasing it (if $\sigma_{j}$ is small). Observe from (16) that we try to reduce the coefficients of (7) on all columns with indices in $B^{*}$ : for continuous variables in $B^{*}$, this should yield a reduction on the resulting cut coefficient; for integer variables, the end result is not so clear because of the integer modularization (cf. end of Section 2), but $\hat{a}_{j}=0$ always results in a zero cut coefficient in the corresponding column. Since we want to reduce the coefficients relative to $B^{*}$ as much as possible, we set $\sigma_{j}=2 \forall j \in\left\{i \in B^{*}: \bar{x}_{i}>0\right\}$, and $\sigma_{j}=1$ otherwise. This prioritizes the reduction of the source row coefficients on the variables with indices $B^{*}$ such that the corresponding component in $\bar{x}$ is nonzero. We experimentally tried other strategies to choose $\sigma_{j}$, but this simple idea turned out to work well in practice. A discussion is given in Section 6. The rest of the R\&S algorithm is unmodified.

Note that this method offers no guarantee of finding a violated cut, nor does it guarantee to increase the cut violation with respect to the cut associated with the original source row. However, R\&S has proven to generate strong cuts in practice, therefore we are interested in testing whether it is equally effective if applied to non-optimal bases of $(\mathrm{LP})$, and in particular those generated by L\&P. 


\section{Combining Lift-and-Project and Reduce-and-Split}

We combined the methods described in Section 3 and Section 4 into a single cut generation algorithm, that alternates between the L\&P and the R\&S cut improvement procedures.

Our cut generation algorithm always starts with determining the set of basic integer variables that have a fractional value (at least $10^{-2}$ away from an integer) in the current solution to the LP relaxation; the corresponding (elementary) disjunctions are processed by nonincreasing violation (i.e. those with a violation closest to 0.5 are processed first), until a given maximum number of cuts $M$ is generated, or there are no more violated disjunctions available. In this paper, we always use $M=50$. This method for processing elementary disjunction is taken from [12]. Recall that these disjunctions give rise to the traditional GMI cuts. Then, we iteratively modify each GMI cut, changing either the underlying disjunction (through R\&S), or the underlying basis of the LP relaxation (through L\&P). One parameter of our cut generation algorithm is the maximum number $\eta$ of cut improvement steps that we want to perform, i.e. the number of times that we alternate between L\&P and R\&S. When $\eta=0$, we use the initial GMI cuts. Another parameter start is whether to apply $L \& P$ or $R \& S$ at the first cut modification step. For instance, if start $=\mathrm{L} \& \mathrm{P}$ and $\eta=3$, the GMI cuts are strengthened by L\&P, then the underlying disjunction is modified by R\&S (using the simplex tableau computed by $\mathrm{L} \& \mathrm{P}$ ), finally we change the basis again (using the new disjunction) with L\&P. After each cut improvement step, we check the outcome of the routine (L\&P or R\&S). If the routine fails, either because it could not improve the cut (i.e. L\&P could not perform improving pivots, or R\&S could not find a disjunction that improves the cut coefficients) or because numerical problems were detected, then the improvement procedure for that particular cut is stopped, and we generate the cut computed at the previous iteration. For instance, for start $=\mathrm{L} \& \mathrm{P}$ and $\eta=3$, if the $\mathrm{R} \& \mathrm{~S}$ algorithm at step 2 fails, we generate the L\&P cut obtained at step 1 . If the cut computed at the previous iteration does not satisfy the numerical requirements, then the cut is discarded and we restart the process with another elementary disjunction. Note that if the first improvement step fails, then we simply generate the initial GMI cut. In all cases, we apply formula (7) to generate the cuts, i.e. we always generate the strengthened disjunctive cuts (7) instead of the simple disjunctive cuts (8).

This method is designed to be balanced between L\&P and R\&S: since we always start with the same $M$ elementary disjunction, we can compare the effects of starting with L\&P or with R\&S. Note that this method is based on simple GMI cuts, which have proved to be one of the most effective and reliable general-purpose classes of cutting planes: our method tries to improve on the GMI cuts, but in case of failure, we revert back to the GMI cuts.

\section{Computational experiments}

The cut generation algorithms presented in this paper were implemented in $\mathrm{C}++$ within the COIN-OR Cgl [16] framework. Our L\&P generator is a modification of the existing CglLandP generator [12]; likewise, the R\&S implementation is based on the existing CglRedSplit2 generator [19]. The CglLandP generator employs advanced simplex algorithm functions, and for this reason it only works with the COIN-OR Clp [17] LP solver. Traditional GMI cuts were generated using the CglLandP generator, setting the maximum number of pivots to zero. We used Cplex 12.1 [21] to perform instance preprocessing and Branch-and-Bound. More details on the interaction between Clp and Cplex are given 
in Section 6.1.

Our set of test instances is a subset of the mixed-integer instances in the union of MIPLIB3 [13], MIPLIB2003 [2] and the set of test instances of the University of Bologna available from http://plato.asu.edu/ftp/unibo/. We selected all mixedinteger instances such that the LP has fewer than 500000 nonzero elements, and such that we were able to generate 10 rounds of cutting planes with the original CglLandP generator in less than 20 minutes. The instance bell5 was not selected because of its poor numerical properties, which made computational experiments give erratic results, thus producing noise in the data instead of useful information. We divide the instances in three difficulty classes, depending on the performance of our Cut-and-Branch algorithm (see Section 6.1) with cutting planes generated by the original CglLandP. Instances are labeled Easy if they can be solved requiring less than one minute of CPU time and 1000 nodes; they are labeled Medium if they are not Easy but can be solved in less than 2 hours; they are Hard if they cannot be solved in 2 hours of total CPU time. A list of instances is given in Table 1. In all tests reported in this section, the value of the optimal solution is given to the solver as a cutoff value so that the time of discovery of integer solutions does not affect the size of the enumeration tree.

\begin{tabular}{|l|l|l|}
\hline Easy & Medium & Hard \\
\hline 10teams & aflow30a & a1c1s1 \\
blend2 & arki001 & aflow40b \\
dcmulti & bell3a & b1c1s1 \\
dsbmip & gesa2 & b2c1s1 \\
egout & gesa2_o & bg512142 \\
fiber & glass4 & dano3mip \\
fixnet6 & mas74 & danoint \\
flugp1 & mas76 & dg012142 \\
gen & misc07 & mkc \\
gesa3 & mod011 & momentum1 \\
gesa3_o & modglob & momentum2 \\
khb05250 & noswot & nsrand-ipx \\
misc06 & pk1 & opt1217 \\
qnet1 & pp08aCUTS & roll3000 \\
qnet1_o & pp08a & set1ch \\
rentacar & qiu & swath \\
rgn & rout & timtab1 \\
vpm1 & tr12-30 \\
vpm2 & \\
\hline
\end{tabular}

Table 1: List of test instances.

\subsection{Cut-and-Branch}

To assess the effectiveness of our cut generation procedure, and compare our cut generator to the traditional L\&P and R\&S cuts, we implemented a Cut-and-Branch algorithm on top of Cplex [21] and Clp [17]. Recall that the L\&P cut generator requires a simplex tableau in Clp format. However, we decided to employ Cplex instead of COIN-OR Cbc as Cut-and-Branch code because of its better reliability. Therefore, we proceed as follows: 
each problem instance is read and preprocessed by Cplex with default settings. The presolved reduced problem is then loaded with Clp, and cutting planes are generated for a maximum of 10 rounds or 20 minutes of CPU time. At each round of cut generation, we perform this sequence of operations. First, we generate at most 50 cuts, and add all of them to the LP formulation. Then, we check if any of the cutting planes generated at previous rounds (and subsequently removed from the LP) is violated by the current fractional point; if so, we add all such cuts to the LP. Finally, the LP is reoptimized, and all inactive cutting planes are removed. The LP formulation obtained after 10 rounds is loaded into Cplex, where another pass of presolve is executed before switching to Branch-and-Bound. To simulate a bare Branch-and-Bound algorithm within the Cplex environment, we apply the following settings:

- Cutting planes are disabled (cutsfactor $=0$, and all cut generation algorithms manually disabled);

- Emphasis on proving optimality (mipemphasis = bestbound);

- Heuristics are disabled (heurfreq $=-1$, and all heuristics manually disabled);

- Absolute and relative integrality gap for optimality set to zero (epgap = 0 , epagap $=0)$.

Constraint and integrality precision were set to $10^{-7}$. All other parameters are left to their default value.

\subsection{Parameters for cut generation}

Our cut generation algorithm is described in Section 5, and has two main parameters: the maximum number $\eta$ of cut improvement steps that we want to perform, and whether to apply L\&P or R\&S at the first cut modification step. Additionally, both the L\&P and the $R \& S$ cut generators require some parameters to perform each improvement step. For L\&P, the pivot selection rule is set to "most negative reduced cost", the maximum number of pivots is set to 10 , and we do not apply the iterative modularization technique discussed in [12]. For R\&S, the maximum support of the disjunction is set to 5, the maximum 1-norm of the disjunction is set to 10, the column selection strategy (i.e. the choice of the set $J_{W}$ ) is set to "first $1 / 3$ of the columns with smallest reduced cost", and the row selection strategy is set to "rows with smallest angle with respect to the source row in the space $J_{W} \cup J_{I}$ " (the latter two parameters correspond to the strategies CS1, R\&S8 in [19]). These parameters were chosen for their performance based on the computational experiences reported in $[12,19]$. Even though other values for the L\&P and R\&S cut generators were tested, for space reason we only report results with this set of values. Our configuration of the L\&P generator is very similar to the default parameters discussed in [12], whereas the R\&S configuration is different than in [19] because in that paper a large number of cuts is generated at each round, but here we want to generate at most 50 cuts per round to facilitate comparisons.

For the combined cut generation algorithm, we tested up to 6 cut improvement iterations, starting either with L\&P or with R\&S. Each combination of parameters yields a different cut generator, which we label as $\mathrm{L} \& \mathrm{P}-\eta$ if $\mathrm{L} \& \mathrm{P}$ is applied first and we perform up to $\eta$ improvement steps alternating between R\&S and L\&P, or as R\&S- $\eta$ if $R \& S$ is applied first and we perform up to $\eta$ improvement steps alternating between L\&P and R\&S. Note that L\&P-1 and R\&S-1 correspond to simple L\&P and R\&S cuts respectively. 
We remark that we performed tests to compare L\&P-1 and R\&S- 1 with the implementations described in [12] and [19]. Computational experiments showed that our L\&P-1 has very similar performance to the original implementation of [12], hence we do not report results with the latter. On the other hand, we found difficult to compare R\&S-1 with the implementation of [19], because of the significant differences in the experimental setup (number of cuts and generation of GMI cuts from the initial tableau). For this reason, we omit a comparison with the results in [19].

\subsection{Results with Cut-and-Branch}

We now report and discuss the results obtained within the framework presented in this section, for several cut generators. For each cut generator and each instance, we report: the amount of integrality gap closed at the root after 10 rounds of cut generation (root gap \%), the CPU time required for cut generation which includes the running of the separation procedures as well as the repeated solutions of the node LPs (cut time), the number of generated cuts (\#cuts), the amount of integrality gap closed at the end of the Cut-and-Branch algorithm (100\% if optimality is proven within the time limit, $<100 \%$ if the two hours limit is hit) ( final gap \%), the number of enumerated nodes (\#nodes), and the total CPU time required by Cut-and-Branch (total time). All times are measured in seconds. Detailed results can be found in Tables 6 through 11, whereas averages are given in Table 2. The average integrality gap and number of cuts are computed as arithmetic averages; the average CPU time and number of nodes are geometric averages (to deal with zero values, we added one to each value before computing the average, and subtracted one from the result). For comparison, we also report, in Tables 3 and 12, results obtained within the same framework using traditional GMI cuts from the optimal tableau.

Table 2: Average values for Tables 6 through 11.

\begin{tabular}{|c|c|c|c|c|c|c|c|c|c|c|c|c|c|}
\hline \multirow[b]{2}{*}{$\eta$} & \multirow[b]{2}{*}{ instances } & \multicolumn{6}{|c|}{$\mathrm{L \& P}$} & \multicolumn{6}{|c|}{ R\&S } \\
\hline & & $\begin{array}{r}\text { root } \\
\text { gap \% }\end{array}$ & $\begin{array}{r}\text { cut } \\
\text { time }\end{array}$ & \#cuts & $\begin{array}{r}\text { final } \\
\text { gap \% }\end{array}$ & \#nodes & $\begin{array}{l}\text { total } \\
\text { time }\end{array}$ & $\begin{array}{r}\text { root } \\
\text { gap \% }\end{array}$ & $\begin{array}{r}\text { cut } \\
\text { time }\end{array}$ & \#cuts & $\begin{array}{r}\text { final } \\
\text { gap \% }\end{array}$ & \#nodes & $\begin{array}{l}\text { total } \\
\text { time }\end{array}$ \\
\hline \multirow{3}{*}{1} & Easy & 66.69 & 1.88 & & 100.00 & & 2.34 & 62.70 & 1.48 & 177.12 & 100.00 & & 1.87 \\
\hline & & & & & & & 27.97 & & & & 100.00 & 1.35 & 25.64 \\
\hline & Harc & .67 & 17.73 & 362.95 & 63.99 & 491855.55 & 7200.02 & 27.07 & 9.02 & 328.26 & 65.86 & 529225.83 & 7200.03 \\
\hline \multirow{3}{*}{2} & Easy & & 2.97 & 239.94 & \begin{tabular}{|l|}
100.00 \\
\end{tabular} & 58.13 & 3.36 & 71.07 & 2.45 & 219.50 & 100.00 & 61.68 & 2.99 \\
\hline & & & 2 & & & & & & 1.67 & & & & 28.96 \\
\hline & $\mathrm{Ha}$ & 7 & 29.68 & 368 & 64.76 & 488767. & 200.03 & 28.76 & 17.71 & 334.21 & 63.17 & 527506.47 & 7200.03 \\
\hline \multirow{3}{*}{3} & & & & & & & & & & & & & 3.92 \\
\hline & & & 12 & & & & & & & & & .14 & 30.80 \\
\hline & Hal & .53 & 33.18 & 89 & 65.42 & 505878.43 & 151.24 & 30.42 & 27.89 & 334.95 & 63.16 & 511616.51 & 200.03 \\
\hline \multirow{3}{*}{4} & & & 3.00 & 25 & 100.00 & & 3.32 & & 3.28 & 215.88 & 100.00 & 49.71 & 3.81 \\
\hline & & & 2.14 & 272.35 & 100.00 & 18443.68 & 26.46 & & 2.03 & & 100.00 & 21863.21 & 29.03 \\
\hline & & .32 & 34.45 & 368.53 & 65.59 & 488861.77 & 080.50 & 30.30 & 29.63 & 332.11 & 63.14 & 489310.36 & 7200.04 \\
\hline \multirow{3}{*}{5} & & & 3.27 & 234.50 & .00 & & & & 3.43 & 219.75 & & 47.71 & 3.94 \\
\hline & & & & & & 3520 & & & 2.08 & & & 22320.37 & 28.25 \\
\hline & & & 38.10 & 362. & & 501422.54 & 7200.03 & 29.40 & 29.93 & 335.42 & 63.48 & 520554.34 & 7200.04 \\
\hline \multirow{3}{*}{6} & & & & & & & & & 3.24 & & & & 3.77 \\
\hline & & & 2.22 & & & & & & 2.10 & & & & 27.81 \\
\hline & Hard & 32.06 & 37.66 & 369.74 & 65.29 & 468780.03 & 7200.04 & 29.99 & 29.76 & 329.26 & 62.84 & 482300.74 & 7200.03 \\
\hline
\end{tabular}

Table 3: Average values for Table 12.

\begin{tabular}{|l|r|r|r|r|r|r|}
\hline & \multicolumn{6}{|c|}{ GMI } \\
\cline { 2 - 7 } instances & $\begin{array}{r}\text { root } \\
\text { gap \% }\end{array}$ & $\begin{array}{r}\text { cut } \\
\text { time }\end{array}$ & \#cuts & $\begin{array}{r}\text { final } \\
\text { gap \% }\end{array}$ & \#nodes & $\begin{array}{r}\text { total } \\
\text { time }\end{array}$ \\
\hline Easy & 52.38 & 0.31 & 197.81 & 100.00 & 104.12 & 0.76 \\
Medium & 36.51 & 0.30 & 256.50 & 100.00 & 47544.46 & 27.02 \\
Hard & 26.45 & 1.86 & 328.63 & 64.33 & 571427.50 & 7200.03 \\
\hline
\end{tabular}


In the integer programming community it is known that comparing the strength of different cut generators is a difficult task, especially when we are interested in the performance of Cut-and-Branch, and average values alone can be misleading. [22] proposes a framework for statistical tests. In this paper, in addition to reporting average values, we opted for a simple pairwise comparison between the 13 cut generators tested; in each comparison, we count the number of instances on which the first method is superior to the second one. The comparison is carried out on Medium and Hard instances, because on Easy instance we expect simple GMI cuts to outperform more powerful but timeconsuming cut generation methods. Our comparison criteria are as follows: method $A$ is superior to method $B$ on a given instance if:

- Medium instance: $A$ solves the instance in $10 \%$ less CPU time than $B$ and the difference is at least 2 seconds, or $A$ and $B$ take approximately the same CPU time ( $\pm 5 \%$ or a difference of less than 2 seconds) and $A$ requires $20 \%$ fewer nodes.

- Hard instance: $A$ closes at least $\rho=1 \%$ more integrality gap than $B$ in the two hours, or $A$ solves the instance within the time limit whereas $B$ does not solve it and has more than $5 \%$ integrality gap left.

On Medium instances, we require a difference of at least 2 seconds of CPU time, because for small values the fluctuations may be due to other factors than the strength of the cutting planes. If no method is superior to the other, then the 2 methods have comparable strength on that instance. We can see from Table 4 that there seem to be an improvement in L\&P- $\eta$ for increasing $\eta$. There is also an improvement in R\&S- $\eta$ for increasing $\eta$, but only for $\eta>2$. From R\&S-1 to R\&S-2, there is a big drop in performance (more on this below). Similarly, on Hard instances, the performance of L\&P- $\eta$ improves as $\eta$ increases from 1 to 5 . In fact, the number of times L\&P- $\eta$ outperforms the other cut generators increases from 75 to 118 , whereas the number of occasions L\&P- $\eta$ is inferior to other methods decreases from 81 to 34 . However, L\&P-6 is inferior to L\&P-5. In Table 5 we report results on Hard instances. We verified that as long as a "reasonable" value for $\rho$ is used $(\rho \in[1,10])$, the conclusions that can be drawn are essentially the same.

We have observed that the number of generated cuts is very similar for all methods, except on Easy instances: for Easy instances, R\&S-1 and GMI generate fewer cuts than other methods. However, our analysis focuses on Medium and Hard instances, and on these two problem classes all algorithms generate a similar number of cuts (the difference can be $\pm 10 \%)$. Therefore, we can compare the cut generators on equal footing.

We can see (Tables 2 and 3) that all proposed methods appear to be stronger than simple GMI cuts in terms of average gap closed at the root node, and in terms of number of nodes for Easy and Medium instances. On Hard instances, the gap closed by GMI cuts after Branch-and-Bound is comparable to some of the other tested method, even though GMI cuts appear to be weaker at the root. However, the number of nodes processed in two hours is larger for GMI cuts, which explains why the amount of gap closed after Branch-and-Bound is similar. GMI cuts are also the fastest method for Easy instances on average, and one of the fastest methods for Medium instances. Table 4 shows that GMI cuts perform well on Medium instances, being "superior" to other methods (but at the same time "inferior") a large number of times. On some hard problems, like danoint and opt1217, GMI cuts are still a good choice (see Table 12), due to a larger number of enumerated nodes within the time limit. This is to be expected: on some problems, investing CPU time in more expensive cut generation techniques does not pay off and GMI cuts come out as the winner, but in other cases, there is a large advantage to be gained by heavy cut generation. 
Table 4: Pairwise comparison of cut generators on Medium instances: number of instances on which the cut generation algorithm on the row is superior to the one on the column. Comparison with $10 \%$ decrease of CPU time or $20 \%$ decrease of number of nodes if CPU time is comparable $( \pm 5 \%)$.

\begin{tabular}{|c|c|c|c|c|c|c|c|c|c|c|c|c|c|c|}
\hline & $\frac{1}{1}$ & 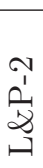 & 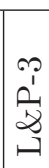 & $\mid \begin{array}{l}-1 \\
\frac{1}{2} \\
3 \\
-1 \\
-1\end{array}$ & $\begin{array}{l}10 \\
2 \\
2 \\
2 \\
-1 \\
-1\end{array}$ & $\begin{array}{l}0 \\
1 \\
1_{1}^{1} \\
3 \\
-1\end{array}$ & $\begin{array}{l}-1 \\
1 \\
2 \\
z \\
\approx 1\end{array}$ & $\begin{array}{c}r \\
1 \\
\tilde{n} \\
3 \\
\tilde{1}\end{array}$ & $\begin{array}{l}n \\
i \\
w \\
z \\
\tilde{z}\end{array}$ & 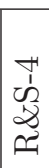 & 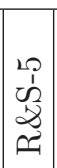 & 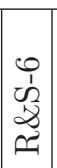 & $\sum_{\mho}$ & 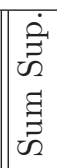 \\
\hline L\&P-1 & - & 4 & 5 & 3 & 6 & 6 & 6 & 6 & 7 & 5 & 5 & 6 & 7 & 66 \\
\hline L\&P-2 & 7 & - & 4 & 4 & 5 & 5 & 8 & 6 & 6 & 5 & 3 & 3 & 7 & 63 \\
\hline L\&P-3 & 6 & 5 & - & 3 & 9 & 9 & 5 & 6 & 7 & 4 & 4 & 5 & 8 & 71 \\
\hline L\&P-4 & 5 & 7 & 6 & - & 7 & 6 & 7 & 9 & 8 & 6 & 7 & 7 & 8 & 83 \\
\hline L\&P-5 & 9 & 7 & 4 & 7 & - & 3 & 8 & 7 & 6 & 5 & 6 & 4 & 8 & 74 \\
\hline L\&P-6 & 6 & 7 & 5 & 5 & 1 & - & 7 & 6 & 4 & 4 & 4 & 5 & 7 & 61 \\
\hline R\&S-1 & 9 & 6 & 8 & 8 & 7 & 8 & - & 8 & 8 & 8 & 7 & 7 & 6 & 90 \\
\hline R\&S-2 & 6 & 3 & 4 & 2 & 6 & 6 & 6 & - & 4 & 3 & 1 & 2 & 6 & 49 \\
\hline R\&S-3 & 7 & 5 & 4 & 4 & 4 & 5 & 5 & 5 & - & 2 & 2 & 2 & 6 & 51 \\
\hline R\&S-4 & 7 & 4 & 5 & 5 & 9 & 8 & 5 & 5 & 5 & - & 3 & 4 & 6 & 66 \\
\hline R\&S-5 & 6 & 6 & 7 & 4 & 7 & 6 & 6 & 5 & 4 & 6 & - & 3 & 6 & 66 \\
\hline R\&S-6 & 6 & 9 & 7 & 4 & 7 & 7 & 6 & 5 & 6 & 5 & 4 & - & 6 & 72 \\
\hline GMI & 8 & 7 & 8 & 7 & 6 & 6 & 5 & 7 & 7 & 8 & 5 & 6 & - & 80 \\
\hline
\end{tabular}

Table 5: Pairwise comparison of cut generators on Hard instances: number of instances on which the cut generation algorithm on the row is superior to the one on the column. Comparison with $1 \%$ absolute difference in final gap closed.

\begin{tabular}{|c|c|c|c|c|c|c|c|c|c|c|c|c|c|c|}
\hline & $\begin{array}{l}7 \\
a^{\prime} \\
2 \\
-1 \\
-1\end{array}$ & 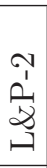 & $\begin{array}{l}n \\
0 \\
\frac{1}{3} \\
-1\end{array}$ & 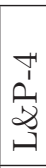 & $\begin{array}{l}10 \\
1 \\
0 \\
2 \\
-1 \\
-1\end{array}$ & $\begin{array}{l}0 \\
1 \\
\rho_{1} \\
3 \\
\ddots\end{array}$ & 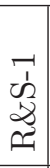 & 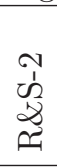 & $\begin{array}{l}0 \\
i \\
\text { in } \\
3 \\
\tilde{n}\end{array}$ & $\begin{array}{c}-1 \\
1 \\
w \\
2 \\
2 \\
\end{array}$ & 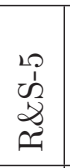 & 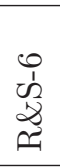 & $\underset{ت}{\mid}$ & 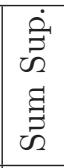 \\
\hline L\&P-1 & - & 4 & 3 & 3 & 2 & 5 & 6 & 8 & 12 & 7 & 9 & 9 & \begin{tabular}{|l|}
7 \\
\end{tabular} & 75 \\
\hline L\&P-2 & 6 & - & 4 & 4 & 2 & 4 & 7 & 8 & 8 & 9 & 10 & 8 & 7 & 77 \\
\hline L\&P-3 & 10 & 7 & - & 6 & 4 & 5 & 8 & 11 & 10 & 9 & 9 & 10 & 10 & 99 \\
\hline L\&P-4 & 9 & 9 & 7 & - & 4 & 5 & 7 & 13 & 10 & 10 & 10 & 12 & 9 & 105 \\
\hline L\&P-5 & 9 & 9 & 7 & 6 & - & 8 & 8 & 11 & 13 & 11 & 14 & 13 & 9 & 118 \\
\hline L\&P-6 & 9 & 9 & 6 & 5 & 2 & - & 8 & 12 & 13 & 11 & 12 & 14 & 10 & 111 \\
\hline R\&S-1 & 6 & 4 & 4 & 6 & 6 & 6 & - & 9 & 7 & 9 & 8 & 8 & 9 & 82 \\
\hline R\&S-2 & 6 & 4 & 3 & 2 & 1 & 3 & 4 & - & 7 & 7 & 6 & 8 & 8 & 59 \\
\hline R\&S-3 & 3 & 6 & 4 & 4 & 1 & 3 & 5 & 6 & - & 5 & 6 & 5 & 6 & 54 \\
\hline R\&S-4 & 7 & 5 & 4 & 1 & 2 & 2 & 6 & 8 & 7 & - & 8 & 5 & 8 & 63 \\
\hline R\&S-5 & 5 & 5 & 3 & 3 & 3 & 2 & 6 & 9 & 7 & 6 & - & 6 & 9 & 64 \\
\hline R\&S-6 & 5 & 4 & 3 & 1 & 2 & 3 & 5 & 6 & 6 & 5 & 5 & - & 7 & 52 \\
\hline GMI & 6 & 7 & 5 & 4 & 5 & 5 & 5 & 7 & 6 & 7 & 8 & 8 & - & 73 \\
\hline um Inf. & 81 & 73 & 53 & 45 & 34 & 51 & 75 & 108 & 106 & 96 & 105 & 106 & 99 & \\
\hline
\end{tabular}


We also observe that R\&S-1 performs very well on average on Medium and especially Hard instances, and appears to be stronger than L\&P-1 by looking at Table 2 only; a more detailed analysis of the results reveals that its good average behaviour depends on some Hard and Medium instances on which R\&S-1 is considerably stronger than other cut generators (examples are bel13a, danoint, dg012412, opt1217, vpm1), but on several other instances R\&S-1 is clearly weaker. This is well indicated by Tables 4 and 5: R\&S-1 is always "inferior" and "superior" a large number of times. We remark that the results in [3] and [19] already showed that reducing cut coefficients with the R\&S algorithm is very strong on some particular instances (e.g. the bell and vpm instances), therefore this is not surprising. It is possible that in these instances, continuous variables play a very important role in terms of the objective function value, which is the ideal setting for R\&S1. R\&S- $\eta$ for $\eta>1$ do not perform equally well as R\&S-1 on the few instances where R\&S-1 really dominates. On average $\eta>1$ yields better results on the Easy and Medium instances in terms of nodes, but not in terms of CPU time, while being comparable on the Hard problems; the gap closed at the root node increases significantly on all problem classes.

The benefits for combining $\mathrm{L} \& \mathrm{P}$ and $\mathrm{R} \& \mathrm{~S}$ are much clearer when improving $\mathrm{L} \& \mathrm{P}$ cuts. Looking at Table 2, L\&P- $\eta$ with $\eta>1$ is superior to L\&P-1 in almost all respects: gap closed at the root (except on Hard instances for some values of $\eta$, for which we observe a slight decrease), number of nodes on the Easy and Medium instances, and gap closed after Branch-and-Bound on the Hard instances. On Medium instances, CPU times for L\&P-2, L\&P-3 and L\&P-4 are better than for L\&P-1, and Table 4 also shows an improvement: when moving from $\eta=1$ to $\eta=6$, the cut generators are "superior" a larger number of times, and "inferior" a smaller number of times. The best value for $\eta$ on Medium instances seems to be $\eta=4$. The same trend is observed on Hard problems, Table 5. The peak is reached by L\&P-5: overall, this generator is the one which is "inferior" the smallest number of times, and is "superior" the largest number of times. Additionally, L\&P-3 and L\&P-4 are the only methods to solve one Hard instance (aflow40b) within the 2 hours time limit; L\&P-4 requires 5240 seconds only for this task. To conclude, L\&P-5 seems to be the best choice for difficult problems in our experiments.

These results suggest that combining the $\mathrm{L} \& \mathrm{P}$ and $\mathrm{R} \& \mathrm{~S}$ algorithms is indeed effective, and that alternating between them $\approx 5$ times yields the best result; after 5 iterations, there is hardly any improvement. Moreover, applying L\&P as the first GMI cut strengthening step seems a better choice than starting with $R \& S$ : this is because $R \& S$ cuts are not as consistently strong as L\&P cuts, being very strong on some problems, but weak on others.

Finally, we observe that the average cut generation time increases by $\approx 50 \%$ from $\eta=1$ to $\eta=2$ : the second step is computationally expensive, but not as expensive as the first one. This is because at each step, we can reuse some of the data computed at previous iterations; in particular, we do not have to recompute the LP basis inverse from scratch. For each $\eta>2$, the CPU time required for cut generation increases by less than $10 \%$, since the number of cuts that are modified decreases. On Easy instances, GMI cuts are the best choice in terms of CPU time, because all other methods spend too much time for cut generation at the root - more than the time needed to solve the instance with GMI cuts only. On Medium instances, all methods improve the root gap closed, and reduce the total number of nodes without deteriorating the CPU time. On Hard instances cut generation is expensive, but it is rewarded by the final gap closed. To conclude, our cuts can be effective in reducing total solution time, provided that we have a mechanism to detect easy instances for which excessive cut generation time is detrimental. This issue is beyond the scope of this paper. 


\subsection{R\&S cuts on non-optimal bases: cut violation}

We provide here a brief analysis of the cut violation when $R \& S$ cuts are generated from non-optimal bases of the LP relaxation. Recall from Section 4 that in this case, we could generate cuts which are not violated, which would have to be discarded. It is natural to ask how often does this happen; this is the question we try to answer in this section. Thus, we recorded the number of non violated cuts that are computed while applying 10 rounds of cuts at the root on our test set, with the L\&P-4 generator (which turned out to be the strongest one, see Section 6.3). We gathered the same statistics for other generators as well, and obtained very similar results, therefore here we only present data for L\&P-4.

As discussed in Section 4, if we generate large cut coefficients on the variables $j \in B^{*}$ which are basic in the optimal LP basis, but are nonbasic in the L\&P basis from which $R \& S$ cuts are generated, then the cut may not be violated. This explains why we modify the R\&S algorithm to try and reduce those cut coefficients as much as possible. How often do we generate non-violated cuts if we employ the R\&S algorithm unmodified? It turns out that, even if we do not consider the set $B^{*}$ when applying the R\&S coefficient reduction algorithm (i.e. we apply the $\mathrm{R} \& \mathrm{~S}$ algorithm directly as described in [19]), only 11 cuts are discarded because they are not violated. This is an extremely small number: for comparison, the total number of generated cuts is 17085 . We give two possible explanations for this behavior. First, the $\mathrm{L} \& \mathrm{P}$ cut given in input to the R\&S procedure cuts off the optimal basic solution $\bar{x}$ by a larger amount than the initial GMI cut; hence, changing the split disjunction to obtain a stronger cut is likely to still cut off $\bar{x}$. Second, sparsity plays in our favor: if the LP tableau on which R\&S is applied is sufficiently sparse, it is likely that computing a linear combination of its rows will not deteriorate the coefficients on the columns $j \in B^{*}$ by a large amount.

Thus, there is not a big margin of improvement for the modification of the R\&S algorithm proposed in Section 4: the number of non-violated cuts is already negligible. Indeed, it turns out that with the modified R\&S algorithm, we still generate 11 nonviolated cuts (in total, we generate 17377 cuts in this case). However, an interesting side effect of the modification is that we close more integrality gap: the average integrality gap closed at the root over all instances after 10 rounds increases from $47.39 \%$ to $49.01 \%$. Hence, the proposed modification seems to have a positive effect. Our intuition is that the modified R\&S algorithm is likely to increase the cut violation, yielding deeper cuts. This can be seen by looking at the expression for the distance cut off (first used as a measure of cut quality in [9]). Suppose the cut is $\alpha x \leq \alpha_{0}$; then the normal vector of the hyperplane represented by this cut is $\alpha$. Therefore, the distance $d$ of the basic solution $\bar{x}$ from the hyperplane $\alpha x=\alpha_{0}$ satisfies $\alpha(\bar{x}+d \alpha)=\alpha_{0}$. From this we get the expression:

$$
d=\left(\alpha_{0}-\alpha \bar{x}\right) /\|\alpha\|_{2}^{2} .
$$

By giving more priority to reducing cut coefficients on the columns $j \in B^{*}$ such that $\bar{x}_{j}>0$, the modified R\&S algorithm acts on both the numerator and the denominator of (17), as opposed to only trying to reduce the denominator.

\subsection{Cut density}

We conclude our computational study with an analysis of the density of the cutting planes generated by the methods proposed in this paper. The density is recorded on all cutting planes generated during the 10 rounds applied at the root node of all instances in our test 
set, and for each cut it is computed as a percentage with respect to the maximum density allowed, i.e.: number of nonzeroes over the maximum number of nonzeroes allowed. The maximum number of nonzeroes allowed is equal to $\min \{n, 1000+n / 5\}$, where $n$ is the number of columns; similar strategies to select the maximum density are used in the Branch-and-Cut solvers COIN-OR Cbc [15] and SCIP [1]. In Figure 4 we report the average density values for all cut generators $\mathrm{L} \& \mathrm{P}-\eta$ and $\mathrm{R} \& \mathrm{~S}-\eta$ with $\eta=1, \ldots, 6$, for each round of cut generation applied at the root. For comparison, we additionally report the same curve for the traditional GMI cuts.

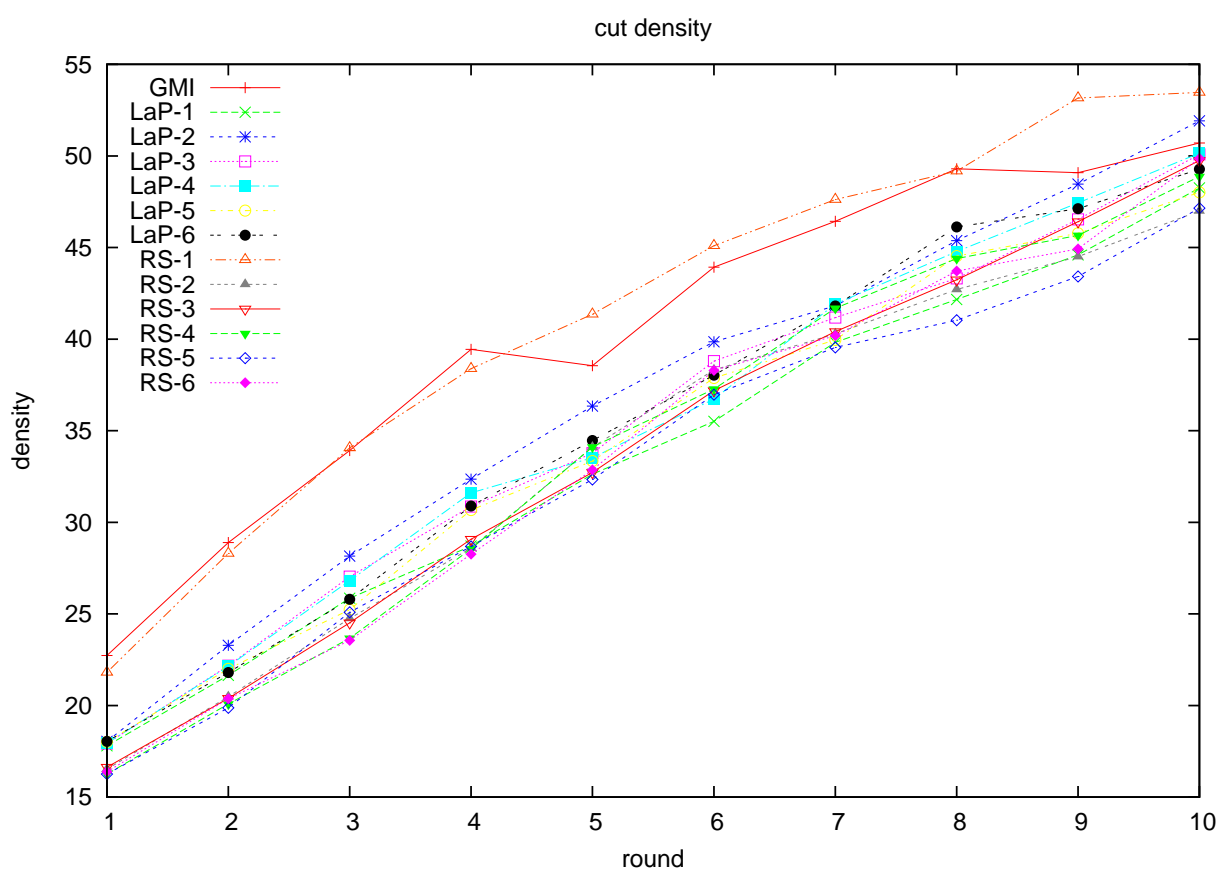

Figure 4: Average cut density for the first 10 rounds.

We can draw some conclusions from the graph. Surprisingly, GMI cuts are the densest cut on average, and they are also denser than most other cuts through the 10 rounds, with the exception of R\&S-1. R\&S-1 is close to GMI in most rounds; therefore, even if it aims at reducing cut coefficients (in the extended $(n+m)$-space, i.e. when the tableau is expressed with equality constraints), it does not reduce density (in the original $n$-space) by a large amount: $\approx 2 \%$ on average. L\&P cuts, on the other hand, appear to be consistently sparser than GMI cuts through all 10 rounds. The same beneficial effect is observed when L\&P and R\&S are combined. An important observation is that there does not seem to be an increase in cut density when $\eta$ moves from 1 to 6 : our combined L\&P $+R \& S$ cut generation algorithm is very stable in this respect, regardless of the number of iterations and whether we start with L\&P or R\&S. Finally, density grows steadily with the number of applied rounds, and the distance between GMI and other cut generators becomes smaller: at the tenth round, all cut generators yield similarly dense cuts, and the density is more than double that of the first round. 


\section{Conclusion}

In this paper we presented a combination of two existing algorithms for generating split cuts: Lift-and-Project and Reduce-and-Split. In doing so, we introduced an extension of the Lift-and-Project procedure on the original simplex tableau that can be employed on general split disjunctions (instead of elementary disjunctions), and we analyzed the application of Reduce-and-Split on non-optimal bases of the LP relaxation. We obtained a cut generation algorithm that iteratively modifies both the LP basis and the split disjunction from which a split cut is generated.

Computational experiments on a set of benchmark instances showed that this combination is effective on mixed-integer instances, solving problems in a smaller number of nodes and closing more integrality gap on the unsolved problems on average. In particular, iterating more than once between $L \& P$ and $R \& S$ proved to be a good choice: in our experiments, applying L\&P first and then iterating 4 times between the two algorithms yielded the best results. Our cut generation algorithm is not significantly slower than the original L\&P and R\&S algorithms, but generates stronger cutting planes that should be useful in practice for the solution of difficult MILPs.

\section{References}

[1] T. Achterberg. Scip: Solving constraint integer programs. Mathematical Programming Computation, 1(1):1-41, 2009.

[2] T. Achterberg, T. Koch, and A. Martin. MIPLIB 2003. Operations Research Letters, 34(4):361-372, 2006.

[3] K. Andersen, G. Cornuéjols, and Y. Li. Reduce-and-split cuts: Improving the performance of mixed integer Gomory cuts. Management Science, 51(11):1720-1732, 2005 .

[4] K. Andersen, G. Cornuéjols, and Y. Li. Split closure and intersection cuts. Mathematical Programming A, 102(3):457-493, 2005.

[5] E. Balas. Intersection cuts - a new type of cutting planes for integer programming. Operations Research, 19(1):19-39, 1971.

[6] E. Balas. Disjunctive programming. Annals of Discrete Mathematics, 5:3-51, 1979.

[7] E. Balas, and R. G. Jeroslow. Stengthening cuts for mixed integer programming European Journal of Operational Research, 4:224-234, 1980.

[8] E. Balas, S. Ceria, and G. Cornuéjols. A lift-and-project cutting plane algorithm for mixed 0-1 programming. Mathematical Programming, 58: 295-324, 1993.

[9] E. Balas, S. Ceria, and G. Cornuéjols. Mixed 0-1 programming by lift-and-project in a branch-and-cut framework. Management Science, 42(9):1229-1246, 1996.

[10] E. Balas, S. Ceria, G. Cornuéjols, and N. Natraj. Gomory cuts revisited. Operations Research Letters, 19(1):1-9, 1996.

[11] E. Balas and M. Perregaard. A precise correspondence between lift-and-project cuts, simple disjunctive cuts, and mixed integer gomory cuts for 0-1 programming. Mathematical Programming, 94(2-3):221-245, 2003. 
[12] E. Balas and P. Bonami. Generating lift-and-project cuts from the lp simplex tableau: open source implementation and testing of new variants. Mathematical Programming Computation, 1:165-199, 2009.

[13] R. E. Bixby, S. Ceria, C. M. McZeal, and M. W. P. Savelsbergh. An updated mixed integer programming library: MIPLIB 3.0. Optima, 58:12-15, 1998.

[14] S. Ceria, G. Cornuéjols, and M. Dawande. Combining and strengthening Gomory cuts. In E. Balas and J. Clausen, editors, Integer Programming and Combinatorial Optimization, volume 920 of Lecture Notes in Computer Science, pages 438-451. Springer Berlin / Heidelberg, 1995.

[15] COIN-OR Branch-and-Cut. https://projects.coin-or.org/Cbc

[16] COIN-OR Cut Generation Library. https://projects.coin-or.org/Cg1

[17] COIN-OR Linear Programming. https://projects.coin-or.org/Clp

[18] W. Cook, R. Kannan, and A. Schrijver. Chvátal closures for mixed integer programming problems. Mathematical Programming, 47:155-174, 1990.

[19] G. Cornuéjols and G. Nannicini. Practical strategies for generating rank-1 split cuts in mixed-integer linear programming. Mathematical Programming Computation, 3:281-318, 2011.

[20] R. E. Gomory. An algorithm for the mixed-integer problem. Technical Report RM-2597, RAND Corporation, 1960.

[21] I. ILOG. IBM ILOG CPLEX 12.1 User's Manual. IBM ILOG, Gentilly, France, 2009.

[22] F. Margot. Testing cut generators for mixed-integer linear programming. Mathematical Programming Computation, 1(1):69-95, 2009.

[23] G. Nemhauser and L. Wolsey. A recursive procedure for generating all cuts for 0-1 mixed integer programs. Mathematical Programming, 46:379-390, 1990.

[24] M. Perregaard. A practical implementation of lift-and-project cuts. In International Symposium on Mathematical Programming, Copenhagen, 2003.

[25] F. Wesselmann. Strengthening Gomory mixed-integer cuts: a computational study. Technical report, University of Paderborn, 2009.

\section{A Detailed tables of results from Section 6.3}


Table 6: Detailed results for L\&P-1 and R\&S-1 cuts.

\begin{tabular}{|c|c|c|c|c|c|c|c|c|c|c|c|c|}
\hline \multirow[b]{2}{*}{ instance } & \multicolumn{6}{|c|}{ L\&P-1 } & \multicolumn{6}{|c|}{ R\&S-1 } \\
\hline & $\begin{array}{r}\text { root } \\
\text { gap \% }\end{array}$ & $\begin{array}{r}\text { cut } \\
\text { time }\end{array}$ & \#cuts & $\begin{array}{r}\text { final } \\
\text { gap } \%\end{array}$ & \#nodes & $\begin{array}{l}\text { total } \\
\text { time }\end{array}$ & $\begin{array}{r}\text { root } \\
\text { gap \% }\end{array}$ & $\begin{array}{r}\text { cut } \\
\text { time }\end{array}$ & \#cuts & $\begin{array}{r}\text { final } \\
\text { gap \% }\end{array}$ & \#nodes & $\begin{array}{l}\text { total } \\
\text { time }\end{array}$ \\
\hline 10teams & 100.00 & 16.54 & 252 & 100.00 & 161 & 21.08 & 100.00 & 8.25 & 4 & 100.00 & 161 & 12.79 \\
\hline blend2 & 32.56 & 0.18 & 129 & 100.00 & 917 & 0.50 & 29.08 & 0.15 & 114 & 100.00 & 929 & 0.42 \\
\hline dcmulti & 86.52 & 2.25 & 398 & 100.00 & 94 & 2.46 & 72.64 & 0.77 & 348 & 100.00 & 209 & 1.09 \\
\hline dsbmip & 0.00 & 4.56 & 384 & 100.00 & 13 & 4.74 & 0.00 & 4.12 & 364 & 100.00 & 13 & 4.30 \\
\hline egout & 100.00 & 0.02 & 55 & 100.00 & 0 & 0.02 & 100.00 & 0.01 & 32 & 100.00 & 0 & 0.01 \\
\hline fiber & 91.77 & 1.68 & 305 & 100.00 & 508 & 2.06 & 88.52 & 4.76 & 206 & 100.00 & 570 & 5.47 \\
\hline fixnet 6 & 56.72 & 1.54 & 189 & 100.00 & 693 & 3.07 & 49.94 & 0.60 & 161 & 100.00 & 376 & 1.06 \\
\hline flugpl & 16.36 & 0.02 & 73 & 100.00 & 290 & 0.03 & 96.88 & 0.01 & 50 & 100.00 & 64 & 0.01 \\
\hline gen & 96.07 & 0.25 & 95 & 100.00 & 2 & 0.26 & 91.65 & 0.15 & 92 & 100.00 & 2 & 0.17 \\
\hline gesa3 & 81.04 & 5.85 & 500 & 100.00 & 94 & 6.11 & 44.11 & 2.91 & 500 & 100.00 & 240 & 3.20 \\
\hline gesa3_o & 89.57 & 5.28 & 403 & 100.00 & 73 & 5.49 & 61.69 & 2.71 & 145 & 100.00 & 312 & 3.06 \\
\hline khb05250 & 99.13 & 0.46 & 106 & 100.00 & 13 & 0.52 & 96.68 & 0.31 & 109 & 100.00 & 22 & 0.37 \\
\hline $\operatorname{misc06}$ & 96.99 & 0.44 & 96 & 100.00 & 12 & 0.50 & 80.30 & 0.29 & 105 & 100.00 & 32 & 0.36 \\
\hline qnet1 & 51.10 & 4.76 & 461 & 100.00 & 585 & 10.78 & 33.05 & 6.14 & 283 & 100.00 & 495 & 8.36 \\
\hline qnet1_o & 69.26 & 3.07 & 405 & 100.00 & 237 & 4.19 & 58.67 & 4.74 & 311 & 100.00 & 527 & 7.91 \\
\hline rentacar & 0.00 & 2.17 & 34 & 100.00 & 17 & 2.52 & 0.00 & 0.93 & 10 & 100.00 & 30 & 1.37 \\
\hline aflow30a & 47.13 & 4.10 & 409 & 100.00 & 25987 & 62.30 & 29.50 & 1.43 & 313 & 100.00 & 54983 & 62.03 \\
\hline arki001 & 50.59 & 7.78 & 408 & 100.00 & 490040 & 1202.43 & 57.06 & 6.81 & 490 & 100.00 & 276275 & 833.40 \\
\hline bell3a & 62.16 & 0.03 & 26 & 100.00 & 27922 & 1.74 & 62.16 & 0.02 & 29 & 100.00 & 15696 & 1.56 \\
\hline gesa2 & 94.74 & 5.53 & 443 & 100.00 & 2662 & 8.04 & 91.62 & 3.68 & 496 & 100.00 & 2274 & 6.25 \\
\hline gesa2_o & 94.28 & 4.20 & 461 & 100.00 & 3822 & 8.19 & 91.17 & 3.42 & 417 & 100.00 & 1148 & 4.93 \\
\hline glass4 & 0.00 & 0.26 & 314 & 100.00 & 463683 & 114.80 & 0.00 & 0.24 & 251 & 100.00 & 2265847 & 515.80 \\
\hline $\operatorname{mas} 74$ & 9.21 & 0.23 & 188 & 100.00 & 2981710 & 319.32 & 8.11 & 0.06 & 87 & 100.00 & 2885266 & 268.69 \\
\hline $\operatorname{mas} 76$ & 9.35 & 0.22 & 168 & 100.00 & 545667 & 48.21 & 8.16 & 0.04 & 62 & 100.00 & 516026 & 40.76 \\
\hline $\operatorname{misc} 07$ & 2.43 & 0.92 & 246 & 100.00 & 39561 & 24.46 & 0.72 & 0.39 & 126 & 100.00 & 18140 & 10.45 \\
\hline $\bmod 011$ & 12.22 & 8.45 & 144 & 100.00 & 6406 & 57.72 & 0.26 & 6.57 & 1 & 100.00 & 17246 & 81.09 \\
\hline modglob & 62.63 & 1.14 & 340 & 100.00 & 92705 & 45.23 & 56.32 & 0.57 & 308 & 100.00 & 171204 & 80.24 \\
\hline noswot & -0.00 & 0.08 & 163 & 100.00 & 694040 & 104.47 & 0.00 & 0.07 & 181 & 100.00 & 694040 & 103.57 \\
\hline $\mathrm{pk} 1$ & 0.00 & 0.07 & 150 & 100.00 & 279380 & 35.56 & 0.00 & 0.07 & 149 & 100.00 & 279380 & 36.01 \\
\hline pp08aCUTS & 87.04 & 1.78 & 385 & 100.00 & 1283 & 3.20 & 73.49 & 0.63 & 418 & 100.00 & 4596 & 4.42 \\
\hline pp08a & 95.03 & 0.75 & 329 & 100.00 & 2106 & 2.13 & 94.93 & 0.33 & 378 & 100.00 & 2883 & 2.43 \\
\hline qiu & 27.94 & 18.71 & 467 & 100.00 & 29448 & 563.77 & 11.44 & 5.04 & 469 & 100.00 & 12591 & 121.07 \\
\hline rgn & 51.36 & 0.08 & 144 & 100.00 & 3580 & 0.42 & 70.66 & 0.06 & 132 & 100.00 & 2262 & 0.32 \\
\hline rout & 31.68 & 2.40 & 454 & 100.00 & 128541 & 203.35 & 10.33 & 0.67 & 269 & 100.00 & 181745 & 135.69 \\
\hline vpm1 & 55.54 & 0.07 & 134 & 100.00 & 2665 & 0.47 & 100.00 & 0.03 & 79 & 100.00 & 1 & 0.04 \\
\hline vpm2 & 63.10 & 0.41 & 288 & 100.00 & 16907 & 5.50 & 51.80 & 0.22 & 294 & 100.00 & 18999 & 6.67 \\
\hline a1c1s1 & 27.21 & 27.27 & 499 & 63.82 & 799673 & 7200.00 & 23.41 & 6.18 & 478 & 61.43 & 1419778 & 7200.01 \\
\hline aflow $40 \mathrm{~b}$ & 34.15 & 14.67 & 218 & 95.50 & 1063047 & 7200.00 & 19.52 & 3.37 & 81 & 91.87 & 1773851 & 7200.00 \\
\hline $\mathrm{b} 1 \mathrm{c} 1 \mathrm{~s} 1$ & 16.80 & 41.81 & 500 & 69.01 & 397488 & 7200.00 & 17.06 & 9.09 & 486 & 69.27 & 599906 & 7200.01 \\
\hline $\mathrm{b} 2 \mathrm{c} 1 \mathrm{~s} 1$ & 16.09 & 59.47 & 500 & 66.58 & 173045 & 7200.01 & 10.24 & 13.28 & 427 & 64.63 & 281404 & 7200.00 \\
\hline bg512142 & 3.00 & 13.79 & 500 & 49.61 & 774056 & 7200.00 & 2.61 & 6.92 & 492 & 49.38 & 745056 & 7200.00 \\
\hline dano3mip & 0.02 & 178.92 & 6 & 1.04 & 2101 & 7200.04 & 0.03 & 61.26 & 7 & 1.06 & 2458 & 7200.04 \\
\hline danoint & 1.16 & 3.50 & 348 & 61.35 & 576748 & 7200.01 & 1.07 & 1.44 & 358 & 79.97 & 750774 & 7200.00 \\
\hline dg012142 & 0.01 & 23.77 & 500 & 49.53 & 561717 & 7200.00 & 0.01 & 31.92 & 498 & 55.71 & 616579 & 7200.01 \\
\hline $\mathrm{mkc}$ & 54.21 & 5.29 & 198 & 81.31 & 2832166 & 7200.00 & 32.28 & 21.89 & 216 & 80.97 & 2407618 & 7200.00 \\
\hline momentum1 & 64.54 & 312.14 & 230 & 65.35 & 521 & 7200.06 & 64.52 & 144.15 & 227 & 81.97 & 527 & 7200.12 \\
\hline momentum2 & 40.77 & 1074.65 & 229 & 68.77 & 477 & 7200.17 & 39.05 & 310.60 & 219 & 68.78 & 488 & 7200.24 \\
\hline nsrand-ipx & 61.80 & 35.88 & 217 & 87.60 & 1434311 & 7200.00 & 49.78 & 9.75 & 108 & 86.34 & 1713539 & 7200.00 \\
\hline opt 1217 & 20.96 & 1.22 & 268 & 22.83 & 4739969 & 7200.08 & 54.19 & 1.20 & 222 & 54.19 & 8413096 & 7200.01 \\
\hline roll3000 & 53.08 & 16.62 & 393 & 82.06 & 952937 & 7200.00 & 7.46 & 6.53 & 289 & 61.47 & 772180 & 7200.00 \\
\hline set1ch & 67.41 & 2.40 & 500 & 93.70 & 10653874 & 7200.00 & 48.02 & 1.17 & 500 & 79.31 & 3831719 & 7200.09 \\
\hline swath & 28.40 & 17.98 & 290 & 58.08 & 896689 & 7200.00 & 28.11 & 10.54 & 129 & 58.71 & 1014968 & 7200.00 \\
\hline timtab1 & 39.72 & 0.74 & 500 & 87.38 & 16443060 & 7199.99 & 42.21 & 0.36 & 500 & 91.82 & 16792769 & 7200.00 \\
\hline timtab2 & 27.08 & 1.32 & 500 & 56.41 & 10903845 & 7200.01 & 26.92 & 1.24 & 500 & 57.18 & 10039227 & 7200.00 \\
\hline $\operatorname{tr} 12-30$ & 45.36 & 4.57 & 500 & 55.86 & 5776355 & 7200.01 & 47.76 & 3.12 & 500 & 57.31 & 3431033 & 7200.00 \\
\hline
\end{tabular}


A DETAILED TABLES OF RESULTS FROM SECTION 6.3

Table 7: Detailed results for L\&P-2 and R\&S-2 cuts.

\begin{tabular}{|c|c|c|c|c|c|c|c|c|c|c|c|c|}
\hline \multirow[b]{2}{*}{ instance } & \multicolumn{6}{|c|}{$\mathrm{L} \& \mathrm{P}-2$} & \multicolumn{6}{|c|}{$\overline{R \& S-2}$} \\
\hline & $\begin{array}{r}\text { root } \\
\text { gap } \%\end{array}$ & $\begin{array}{r}\text { cut } \\
\text { time }\end{array}$ & \#cuts & $\begin{array}{r}\text { final } \\
\text { gap } \%\end{array}$ & \#nodes & $\begin{array}{l}\text { total } \\
\text { time }\end{array}$ & $\begin{array}{r}\text { root } \\
\text { gap } \%\end{array}$ & $\begin{array}{r}\text { cut } \\
\text { time }\end{array}$ & \#cuts & $\begin{array}{r}\text { final } \\
\text { gap } \%\end{array}$ & \#nodes & $\begin{array}{l}\text { total } \\
\text { time }\end{array}$ \\
\hline 10teams & 100.00 & 35.83 & 213 & 100.00 & 161 & 40.37 & 100.00 & 7.36 & 4 & 100.00 & 161 & 11.89 \\
\hline blend2 & 35.83 & 0.47 & 147 & 100.00 & 917 & 0.75 & 32.53 & 0.38 & 146 & 100.00 & 908 & 0.70 \\
\hline dcmulti & 87.44 & 4.00 & 440 & 100.00 & 107 & 4.35 & 82.87 & 2.63 & 399 & 100.00 & 210 & 3.03 \\
\hline dsbmip & 0.00 & 6.03 & 350 & 100.00 & 13 & 6.22 & 0.00 & 6.95 & 375 & 100.00 & 13 & 7.14 \\
\hline egout & 100.00 & 0.02 & 47 & 100.00 & 0 & 0.02 & 100.00 & 0.01 & 32 & 100.00 & 0 & 0.01 \\
\hline fiber & 89.82 & 4.44 & 322 & 100.00 & 570 & 5.15 & 92.35 & 5.73 & 257 & 100.00 & 515 & 6.12 \\
\hline fixnet 6 & 57.92 & 1.67 & 167 & 100.00 & 605 & 3.17 & 52.95 & 1.68 & 197 & 100.00 & 1053 & 4.25 \\
\hline flugpl & 75.47 & 0.01 & 67 & 100.00 & 133 & 0.02 & 98.86 & 0.01 & 71 & 100.00 & 28 & 0.02 \\
\hline gen & 96.94 & 0.35 & 85 & 100.00 & 0 & 0.37 & 94.31 & 0.35 & 98 & 100.00 & 0 & 0.37 \\
\hline gesa3 & 70.22 & 9.98 & 500 & 100.00 & 183 & 10.41 & 75.19 & 8.93 & 500 & 100.00 & 76 & 9.26 \\
\hline gesa3_o & 90.06 & 9.25 & 404 & 100.00 & 34 & 9.43 & 93.05 & 7.19 & 346 & 100.00 & 43 & 7.29 \\
\hline khb05250 & 98.66 & 0.58 & 99 & 100.00 & 16 & 0.64 & 97.07 & 0.61 & 105 & 100.00 & 18 & 0.67 \\
\hline misc06 & 99.73 & 0.56 & 95 & 100.00 & 7 & 0.61 & 94.28 & 0.57 & 112 & 100.00 & 22 & 0.62 \\
\hline qnet1 & 50.15 & 12.44 & 468 & 100.00 & 449 & 14.84 & 49.93 & 11.21 & 463 & 100.00 & 598 & 16.86 \\
\hline qnet1_o & 70.65 & 6.77 & 401 & 100.00 & 301 & 7.81 & 73.77 & 5.90 & 396 & 100.00 & 300 & 7.27 \\
\hline rentacar & 0.00 & 2.46 & 34 & 100.00 & 17 & 2.80 & 0.00 & 1.75 & 11 & 100.00 & 30 & 2.18 \\
\hline aflow30a & 47.17 & 5.79 & 398 & 100.00 & 32231 & 60.44 & 43.15 & 4.58 & 401 & 100.00 & 31300 & 62.41 \\
\hline arki001 & 55.89 & 14.21 & 463 & 100.00 & 195454 & 634.03 & 56.51 & 8.89 & 487 & 100.00 & 230492 & 552.87 \\
\hline bel13a & 62.16 & 0.03 & 30 & 100.00 & 28082 & 1.71 & 62.16 & 0.03 & 26 & 100.00 & 24756 & 1.54 \\
\hline gesa2 & 97.58 & 9.24 & 478 & 100.00 & 236 & 9.58 & 96.98 & 8.10 & 483 & 100.00 & 157 & 8.36 \\
\hline gesa2_o & 97.41 & 7.86 & 454 & 100.00 & 680 & 8.56 & 98.41 & 6.63 & 446 & 100.00 & 115 & 6.77 \\
\hline glass4 & 0.00 & 0.27 & 314 & 100.00 & 463683 & 113.37 & 0.00 & 0.32 & 305 & 100.00 & 2265847 & 520.24 \\
\hline $\operatorname{mas} 74$ & 9.02 & 0.29 & 178 & 100.00 & 3012675 & 315.93 & 8.69 & 0.20 & 132 & 100.00 & 3155390 & 354.51 \\
\hline $\operatorname{mas} 76$ & 9.08 & 0.23 & 146 & 100.00 & 494158 & 40.92 & 9.36 & 0.12 & 122 & 100.00 & 465009 & 40.84 \\
\hline $\operatorname{misc} 07$ & 1.61 & 1.22 & 278 & 100.00 & 16243 & 10.58 & 4.05 & 1.05 & 282 & 100.00 & 46806 & 28.24 \\
\hline $\bmod 011$ & 5.19 & 13.09 & 131 & 100.00 & 13603 & 81.42 & 0.28 & 9.35 & 3 & 100.00 & 19128 & 97.00 \\
\hline modglob & 65.22 & 1.38 & 312 & 100.00 & 17820 & 9.67 & 63.27 & 1.75 & 368 & 100.00 & 47297 & 29.91 \\
\hline noswot & -0.00 & 0.09 & 152 & 100.00 & 694040 & 104.12 & 0.00 & 0.11 & 192 & 100.00 & 694040 & 104.29 \\
\hline pk1 & 0.00 & 0.07 & 150 & 100.00 & 279380 & 35.65 & 0.00 & 0.07 & 149 & 100.00 & 279380 & 36.01 \\
\hline pp08aCUTS & 90.09 & 1.94 & 344 & 100.00 & 1938 & 4.38 & 89.85 & 1.66 & 361 & 100.00 & 1646 & 3.84 \\
\hline pp08a & 95.76 & 1.20 & 353 & 100.00 & 1679 & 2.74 & 96.51 & 0.77 & 327 & 100.00 & 1440 & 1.96 \\
\hline qiu & 28.13 & 18.77 & 467 & 100.00 & 48408 & 796.39 & 27.71 & 22.07 & 469 & 100.00 & 58757 & 1079.17 \\
\hline rgn & 57.61 & 0.09 & 138 & 100.00 & 4114 & 0.57 & 66.36 & 0.08 & 128 & 100.00 & 3589 & 0.44 \\
\hline rout & 33.21 & 4.07 & 458 & 100.00 & 214771 & 356.08 & 31.60 & 2.62 & 442 & 100.00 & 123222 & 235.92 \\
\hline vpm1 & 78.19 & 0.05 & 88 & 100.00 & 112 & 0.08 & 92.99 & 0.04 & 79 & 100.00 & 39 & 0.06 \\
\hline vpm2 & 68.74 & 0.65 & 302 & 100.00 & 5125 & 2.67 & 58.98 & 0.53 & 310 & 100.00 & 5640 & 2.50 \\
\hline a1c1s1 & 23.71 & 42.54 & 497 & 60.44 & 814055 & 7200.00 & 26.28 & 29.11 & 457 & 62.35 & 954268 & 7200.01 \\
\hline aflow $40 \mathrm{~b}$ & 34.61 & 18.72 & 255 & 94.92 & 1443596 & 7200.00 & 22.03 & 4.45 & 87 & 98.21 & 2025478 & 7200.00 \\
\hline $\mathrm{b} 1 \mathrm{c} 1 \mathrm{~s} 1$ & 18.05 & 67.57 & 500 & 68.56 & 381824 & 7200.02 & 15.01 & 34.61 & 444 & 66.81 & 421127 & 7200.02 \\
\hline b2c1s 1 & 16.98 & 99.04 & 500 & 67.26 & 156537 & 7200.01 & 14.61 & 52.50 & 404 & 63.13 & 185771 & 7200.01 \\
\hline bg512142 & 2.97 & 28.45 & 500 & 48.44 & 544550 & 7200.01 & 2.27 & 16.40 & 496 & 49.90 & 608810 & 7199.99 \\
\hline dano3mip & 0.02 & 418.07 & 6 & 1.02 & 1881 & 7200.01 & 0.03 & 93.50 & 7 & 1.06 & 2373 & 7200.13 \\
\hline danoint & 1.16 & 5.73 & 354 & 81.80 & 685112 & 7200.00 & 1.33 & 3.76 & 352 & 66.54 & 583194 & 7200.01 \\
\hline dg012142 & 0.01 & 73.97 & 500 & 52.31 & 555966 & 7200.01 & 0.01 & 46.56 & 494 & 52.74 & 530445 & 7200.01 \\
\hline $\mathrm{mkc}$ & 41.92 & 15.53 & 222 & 80.41 & 2590007 & 7200.00 & 63.57 & 14.97 & 235 & 81.21 & 2490173 & 7200.00 \\
\hline momentum1 & 64.49 & 396.92 & 266 & 64.75 & 489 & 7200.15 & 62.06 & 344.01 & 220 & 64.67 & 514 & 7200.19 \\
\hline momentum2 & 40.92 & 1043.20 & 195 & 68.80 & 506 & 7200.28 & 39.57 & 1042.54 & 230 & 68.84 & 512 & 7200.15 \\
\hline nsrand-ipx & 62.97 & 47.95 & 270 & 92.60 & 1772810 & 7200.00 & 50.21 & 11.47 & 105 & 84.04 & 1731898 & 7200.00 \\
\hline opt 1217 & 17.60 & 1.38 & 267 & 24.41 & 11270792 & 7200.01 & 13.78 & 0.83 & 224 & 21.77 & 10414613 & 7200.00 \\
\hline roll3000 & 28.89 & 28.08 & 413 & 70.92 & 665477 & 7200.01 & 39.53 & 19.37 & 403 & 83.16 & 1014449 & 7200.00 \\
\hline set $1 \mathrm{ch}$ & 63.64 & 4.73 & 499 & 87.61 & 6815028 & 7200.00 & 56.50 & 3.76 & 500 & 87.57 & 6527500 & 7200.01 \\
\hline swath & 28.43 & 21.18 & 273 & 58.93 & 1062400 & 7200.01 & 27.39 & 18.73 & 192 & 49.51 & 981648 & 7200.01 \\
\hline timtab1 & 48.84 & 1.82 & 497 & 92.65 & 16375094 & 7200.00 & 45.70 & 0.97 & 500 & 90.96 & 16663797 & 7200.00 \\
\hline timtab2 & 31.81 & 3.96 & 494 & 58.96 & 7914985 & 7200.00 & 27.71 & 1.99 & 500 & 58.30 & 9757182 & 7200.00 \\
\hline $\operatorname{tr} 12-30$ & 46.16 & 10.72 & 495 & 55.64 & 5626807 & 7200.00 & 38.78 & 7.59 & 500 & 49.43 & 6222719 & 7200.00 \\
\hline
\end{tabular}


A DETAILED TABLES OF RESULTS FROM SECTION 6.3

Table 8: Detailed results for L\&P-3 and R\&S-3 cuts.

\begin{tabular}{|c|c|c|c|c|c|c|c|c|c|c|c|c|}
\hline \multirow[b]{2}{*}{ instance } & \multicolumn{6}{|c|}{$\mathrm{L} \& \mathrm{P}-3$} & \multicolumn{6}{|c|}{ R\&S-3 } \\
\hline & $\begin{array}{r}\text { root } \\
\text { gap } \%\end{array}$ & $\begin{array}{r}\text { cut } \\
\text { time }\end{array}$ & \#cuts & $\begin{array}{r}\text { final } \\
\text { gap } \%\end{array}$ & \#nodes & $\begin{array}{l}\text { total } \\
\text { time }\end{array}$ & $\begin{array}{r}\text { root } \\
\text { gap } \%\end{array}$ & $\begin{array}{r}\text { cut } \\
\text { time }\end{array}$ & \#cuts & $\begin{array}{r}\text { final } \\
\text { gap \% }\end{array}$ & \#nodes & $\begin{array}{l}\text { total } \\
\text { time }\end{array}$ \\
\hline 10teams & 100.00 & 36.81 & 227 & 100.00 & 161 & 41.37 & 100.00 & 9.93 & 4 & 100.00 & 161 & 14.47 \\
\hline blend2 & 34.75 & 0.42 & 134 & 100.00 & 967 & 0.76 & 33.21 & 0.54 & 146 & 100.00 & 935 & 0.82 \\
\hline dcmulti & 89.14 & 3.65 & 412 & 100.00 & 78 & 3.91 & 83.35 & 4.34 & 444 & 100.00 & 158 & 4.68 \\
\hline dsbmip & 0.00 & 6.59 & 354 & 100.00 & 13 & 6.78 & 0.00 & 10.41 & 345 & 100.00 & 13 & 10.60 \\
\hline egout & 100.00 & 0.01 & 44 & 100.00 & 0 & 0.02 & 100.00 & 0.01 & 20 & 100.00 & 0 & 0.01 \\
\hline fiber & 89.89 & 2.51 & 212 & 100.00 & 540 & 2.89 & 90.64 & 10.34 & 286 & 100.00 & 495 & 10.86 \\
\hline fixnet 6 & 59.11 & 2.16 & 182 & 100.00 & 628 & 3.26 & 56.05 & 1.93 & 166 & 100.00 & 1061 & 3.45 \\
\hline flugpl & 97.54 & 0.01 & 57 & 100.00 & 18 & 0.01 & 99.80 & 0.01 & 66 & 100.00 & 3 & 0.02 \\
\hline gen & 97.38 & 0.24 & 64 & 100.00 & 2 & 0.26 & 58.35 & 0.50 & 107 & 100.00 & 2 & 0.52 \\
\hline gesa3 & 78.52 & 10.01 & 500 & 100.00 & 110 & 10.32 & 81.32 & 13.53 & 500 & 100.00 & 51 & 13.78 \\
\hline gesa3_o & 94.93 & 9.78 & 402 & 100.00 & 28 & 9.97 & 89.38 & 11.88 & 377 & 100.00 & 47 & 12.04 \\
\hline khb05250 & 98.79 & 0.55 & 90 & 100.00 & 15 & 0.60 & 98.10 & 0.97 & 114 & 100.00 & 20 & 1.05 \\
\hline misc06 & 99.48 & 0.50 & 89 & 100.00 & 10 & 0.55 & 98.40 & 0.85 & 125 & 100.00 & 12 & 0.90 \\
\hline qnet1 & 47.68 & 12.50 & 461 & 100.00 & 640 & 17.62 & 58.53 & 18.52 & 349 & 100.00 & 286 & 19.84 \\
\hline qnet1_o & 71.34 & 8.19 & 414 & 100.00 & 429 & 9.71 & 74.90 & 11.64 & 415 & 100.00 & 237 & 12.76 \\
\hline rentacar & 0.00 & 2.51 & 34 & 100.00 & 17 & 2.85 & 0.00 & 2.13 & 11 & 100.00 & 30 & 2.57 \\
\hline aflow30a & 44.86 & 6.83 & 393 & 100.00 & 32341 & 73.04 & 43.78 & 6.74 & 385 & 100.00 & 36989 & 75.98 \\
\hline arki001 & 53.49 & 14.12 & 442 & 100.00 & 228453 & 880.62 & 63.45 & 12.66 & 472 & 100.00 & 168103 & 571.50 \\
\hline bel13a & 62.16 & 0.03 & 29 & 100.00 & 33524 & 2.11 & 62.16 & 0.03 & 26 & 100.00 & 24756 & 1.54 \\
\hline gesa2 & 97.37 & 9.89 & 443 & 100.00 & 260 & 10.20 & 96.65 & 13.05 & 492 & 100.00 & 886 & 14.05 \\
\hline gesa2_o & 97.66 & 9.25 & 447 & 100.00 & 578 & 10.03 & 96.28 & 12.18 & 471 & 100.00 & 439 & 12.66 \\
\hline glass4 & 0.00 & 0.27 & 314 & 100.00 & 463683 & 114.52 & 0.00 & 0.34 & 305 & 100.00 & 2265847 & 523.23 \\
\hline $\operatorname{mas} 74$ & 8.92 & 0.34 & 181 & 100.00 & 3013313 & 336.47 & 8.74 & 0.25 & 147 & 100.00 & 4350042 & 488.91 \\
\hline $\operatorname{mas} 76$ & 9.31 & 0.22 & 168 & 100.00 & 540241 & 44.29 & 9.80 & 0.16 & 120 & 100.00 & 496915 & 42.31 \\
\hline $\operatorname{misc} 07$ & 2.51 & 1.15 & 259 & 100.00 & 23080 & 14.97 & 2.62 & 1.34 & 262 & 100.00 & 30760 & 21.35 \\
\hline $\bmod 011$ & 5.31 & 17.26 & 115 & 100.00 & 10769 & 89.92 & 0.28 & 9.45 & 3 & 100.00 & 19128 & 87.36 \\
\hline modglob & 63.57 & 1.92 & 322 & 100.00 & 34601 & 17.67 & 68.94 & 1.71 & 330 & 100.00 & 40151 & 24.69 \\
\hline noswot & -0.00 & 0.10 & 175 & 100.00 & 694040 & 104.50 & -0.00 & 0.13 & 179 & 100.00 & 694040 & 105.24 \\
\hline pk1 & 0.00 & 0.07 & 150 & 100.00 & 279380 & 35.78 & 0.00 & 0.07 & 149 & 100.00 & 279380 & 36.03 \\
\hline pp08aCUTS & 85.40 & 2.66 & 358 & 100.00 & 1669 & 4.25 & 90.87 & 1.54 & 322 & 100.00 & 2090 & 3.82 \\
\hline pp08a & 97.17 & 1.05 & 319 & 100.00 & 992 & 2.28 & 96.34 & 0.75 & 259 & 100.00 & 1180 & 1.71 \\
\hline qiu & 28.62 & 23.10 & 467 & 100.00 & 37797 & 663.80 & 30.82 & 23.66 & 466 & 100.00 & 36000 & 662.47 \\
\hline rgn & 52.66 & 0.17 & 165 & 100.00 & 4059 & 0.65 & 68.50 & 0.09 & 124 & 100.00 & 2956 & 0.46 \\
\hline rout & 44.05 & 4.56 & 434 & 100.00 & 123978 & 243.45 & 40.58 & 4.09 & 434 & 100.00 & 248505 & 597.18 \\
\hline vpm1 & 92.95 & 0.05 & 71 & 100.00 & 54 & 0.06 & 92.99 & 0.06 & 79 & 100.00 & 39 & 0.07 \\
\hline vpm2 & 61.64 & 0.61 & 287 & 100.00 & 3179 & 1.67 & 66.79 & 0.58 & 306 & 100.00 & 3927 & 1.71 \\
\hline a1c1s1 & 28.00 & 53.86 & 499 & 66.52 & 903983 & 7200.01 & 24.03 & 38.14 & 430 & 61.11 & 875210 & 7200.00 \\
\hline aflow $40 \mathrm{~b}$ & 35.13 & 22.23 & 211 & 100.00 & 1471422 & 6327.53 & 26.72 & 5.75 & 109 & 92.11 & 1554640 & 7200.00 \\
\hline $\mathrm{b} 1 \mathrm{c} 1 \mathrm{~s} 1$ & 21.15 & 63.84 & 500 & 71.44 & 364426 & 7200.02 & 15.39 & 56.61 & 409 & 68.64 & 451821 & 7200.00 \\
\hline $\mathrm{b} 2 \mathrm{c} 1 \mathrm{~s} 1$ & 17.31 & 119.59 & 500 & 66.67 & 178552 & 7200.00 & 17.99 & 77.88 & 459 & 62.61 & 166794 & 7200.01 \\
\hline bg512142 & 3.04 & 31.13 & 500 & 49.02 & 579253 & 7200.01 & 2.47 & 32.69 & 489 & 50.46 & 627360 & 7200.01 \\
\hline dano3mip & 0.02 & 404.32 & 6 & 1.04 & 2115 & 7200.08 & 0.03 & 130.95 & 7 & 1.02 & 1905 & 7200.09 \\
\hline danoint & 1.16 & 6.33 & 357 & 63.96 & 576663 & 7200.01 & 1.08 & 5.61 & 354 & 59.28 & 518942 & 7200.01 \\
\hline dg012142 & 0.01 & 79.07 & 500 & 41.48 & 592802 & 7200.00 & 0.01 & 104.81 & 489 & 45.92 & 579770 & 7200.00 \\
\hline $\mathrm{mkc}$ & 50.56 & 22.73 & 257 & 81.00 & 2466335 & 7200.00 & 52.87 & 36.36 & 235 & 79.99 & 2669203 & 7200.00 \\
\hline momentum1 & 64.46 & 373.26 & 259 & 82.23 & 681 & 7200.11 & 64.52 & 560.36 & 234 & 82.36 & 1473 & 7200.09 \\
\hline momentum2 & 32.36 & 1135.28 & 235 & 68.82 & 499 & 7200.18 & 35.97 & 1217.84 & 165 & 68.81 & 487 & 7200.34 \\
\hline nsrand-ipx & 65.75 & 53.89 & 258 & 94.08 & 1901215 & 7200.01 & 56.86 & 14.60 & 119 & 84.39 & 1667384 & 7200.00 \\
\hline opt 1217 & 21.65 & 2.32 & 304 & 24.80 & 10641431 & 7200.00 & 17.86 & 2.37 & 303 & 21.73 & 8138058 & 7200.00 \\
\hline roll3000 & 35.99 & 26.13 & 414 & 83.28 & 1196977 & 7200.00 & 47.06 & 31.44 & 421 & 77.48 & 656312 & 7200.01 \\
\hline set $1 \mathrm{ch}$ & 56.82 & 5.58 & 496 & 85.01 & 6117535 & 7200.05 & 60.11 & 5.65 & 497 & 89.03 & 6954290 & 7200.01 \\
\hline swath & 28.43 & 24.25 & 290 & 58.00 & 931899 & 7200.01 & 27.97 & 19.16 & 148 & 47.16 & 994738 & 7200.00 \\
\hline timtab1 & 43.36 & 2.24 & 492 & 92.07 & 15276320 & 7200.00 & 51.21 & 1.90 & 498 & 93.70 & 15326395 & 7200.01 \\
\hline timtab2 & 31.25 & 4.16 & 494 & 60.75 & 8343697 & 7200.00 & 33.56 & 5.15 & 500 & 61.64 & 8928425 & 7200.02 \\
\hline $\operatorname{tr} 12-30$ & 43.60 & 12.12 & 494 & 52.83 & 4437638 & 7200.00 & 42.36 & 13.57 & 498 & 52.57 & 4942071 & 7200.01 \\
\hline
\end{tabular}


A DETAILED TABLES OF RESULTS FROM SECTION 6.3

Table 9: Detailed results for L\&P-4 and R\&S-4 cuts.

\begin{tabular}{|c|c|c|c|c|c|c|c|c|c|c|c|c|}
\hline \multirow[b]{2}{*}{ instance } & \multicolumn{6}{|c|}{$\mathrm{L} \& \mathrm{P}-4$} & \multicolumn{6}{|c|}{ R\&S-4 } \\
\hline & $\begin{array}{r}\text { root } \\
\text { gap \% }\end{array}$ & $\begin{array}{r}\text { cut } \\
\text { time }\end{array}$ & \#cuts & $\begin{array}{r}\text { final } \\
\text { gap \% }\end{array}$ & \#nodes & $\begin{array}{c}\text { total } \\
\text { time }\end{array}$ & $\begin{array}{r}\text { root } \\
\text { gap \% }\end{array}$ & $\begin{array}{r}\text { cut } \\
\text { time }\end{array}$ & \#cuts & $\begin{array}{r}\text { final } \\
\text { gap \% }\end{array}$ & \#nodes & $\begin{array}{l}\text { total } \\
\text { time }\end{array}$ \\
\hline 10teams & 100.00 & 36.33 & 227 & 100.00 & 161 & 40.85 & 100.00 & 7.49 & 4 & 100.00 & 161 & 12.00 \\
\hline blend2 & 38.55 & 0.52 & 141 & 100.00 & 1055 & 0.85 & 35.43 & 0.54 & 154 & 100.00 & 1020 & 0.87 \\
\hline dcmulti & 89.92 & 3.98 & 422 & 100.00 & 90 & 4.22 & 85.17 & 4.02 & 405 & 100.00 & 123 & 4.36 \\
\hline dsbmip & 0.00 & 6.81 & 367 & 100.00 & 13 & 7.00 & 0.00 & 9.19 & 351 & 100.00 & 13 & 9.38 \\
\hline egout & 100.00 & 0.01 & 44 & 100.00 & 0 & 0.02 & 100.00 & 0.01 & 20 & 100.00 & 0 & 0.01 \\
\hline fiber & 88.45 & 2.49 & 185 & 100.00 & 524 & 2.96 & 92.26 & 8.96 & 249 & 100.00 & 492 & 9.39 \\
\hline fixnet 6 & 59.28 & 1.75 & 160 & 100.00 & 610 & 2.86 & 56.68 & 2.11 & 179 & 100.00 & 702 & 4.03 \\
\hline flugpl & 97.55 & 0.01 & 57 & 100.00 & 16 & 0.01 & 99.93 & 0.02 & 70 & 100.00 & 4 & 0.02 \\
\hline gen & 97.50 & 0.24 & 64 & 100.00 & 2 & 0.25 & 60.76 & 0.49 & 107 & 100.00 & 0 & 0.51 \\
\hline gesa3 & 75.12 & 11.05 & 500 & 100.00 & 71 & 11.30 & 79.46 & 13.18 & 500 & 100.00 & 94 & 13.52 \\
\hline gesa3_o & 90.22 & 8.37 & 374 & 100.00 & 32 & 8.50 & 94.49 & 10.56 & 349 & 100.00 & 29 & 10.67 \\
\hline khb05250 & 98.79 & 0.55 & 90 & 100.00 & 14 & 0.59 & 98.92 & 0.82 & 104 & 100.00 & 15 & 0.89 \\
\hline misc06 & 98.90 & 0.55 & 88 & 100.00 & 9 & 0.60 & 98.48 & 0.82 & 123 & 100.00 & 13 & 0.87 \\
\hline qnet1 & 51.20 & 13.71 & 453 & 100.00 & 371 & 15.23 & 50.20 & 20.52 & 412 & 100.00 & 368 & 22.91 \\
\hline qnet1_o & 75.19 & 10.33 & 398 & 100.00 & 235 & 11.60 & 73.57 & 10.62 & 416 & 100.00 & 525 & 14.42 \\
\hline rentacar & 0.00 & 2.48 & 34 & 100.00 & 17 & 2.83 & 0.00 & 1.95 & 11 & 100.00 & 30 & 2.38 \\
\hline aflow30a & 48.87 & 6.66 & 382 & 100.00 & 26691 & 64.72 & 45.55 & 7.05 & 405 & 100.00 & 29912 & 72.94 \\
\hline arki001 & 63.33 & 12.23 & 424 & 100.00 & 105960 & 337.49 & 61.47 & 12.51 & 475 & 100.00 & 258489 & 670.37 \\
\hline bell3a & 62.16 & 0.03 & 29 & 100.00 & 33524 & 2.11 & 62.16 & 0.03 & 26 & 100.00 & 24756 & 1.55 \\
\hline gesa2 & 97.02 & 10.44 & 445 & 100.00 & 297 & 10.80 & 97.34 & 12.66 & 490 & 100.00 & 263 & 13.02 \\
\hline gesa2_o & 97.73 & 9.62 & 454 & 100.00 & 345 & 9.94 & 96.65 & 11.39 & 444 & 100.00 & 412 & 11.80 \\
\hline glass4 & 0.00 & 0.28 & 314 & 100.00 & 463683 & 115.96 & 0.00 & 0.35 & 305 & 100.00 & 2265847 & 518.50 \\
\hline $\operatorname{mas} 74$ & 8.84 & 0.35 & 176 & 100.00 & 3916510 & 448.68 & 8.81 & 0.26 & 148 & 100.00 & 3159428 & 341.59 \\
\hline $\operatorname{mas} 76$ & 8.99 & 0.28 & 143 & 100.00 & 502255 & 43.56 & 9.47 & 0.13 & 101 & 100.00 & 475130 & 40.81 \\
\hline $\operatorname{misc} 07$ & 2.51 & 1.19 & 245 & 100.00 & 43040 & 24.12 & 1.97 & 1.38 & 285 & 100.00 & 24364 & 15.53 \\
\hline $\bmod 011$ & 5.31 & 19.17 & 114 & 100.00 & 10823 & 93.39 & 0.28 & 9.43 & 3 & 100.00 & 19128 & 87.64 \\
\hline modglob & 67.85 & 2.14 & 327 & 100.00 & 53342 & 30.80 & 74.86 & 1.63 & 314 & 100.00 & 22947 & 12.78 \\
\hline noswot & -0.00 & 0.09 & 179 & 100.00 & 694040 & 104.54 & 0.00 & 0.12 & 168 & 100.00 & 694040 & 105.29 \\
\hline $\mathrm{pk} 1$ & 0.00 & 0.07 & 150 & 100.00 & 279380 & 35.92 & 0.00 & 0.07 & 149 & 100.00 & 279380 & 36.17 \\
\hline pp08aCUTS & 90.89 & 2.54 & 343 & 100.00 & 1186 & 3.82 & 90.58 & 1.72 & 317 & 100.00 & 1361 & 3.28 \\
\hline pp08a & 96.91 & 1.23 & 332 & 100.00 & 1074 & 2.46 & 96.33 & 1.16 & 326 & 100.00 & 1111 & 2.18 \\
\hline qiu & 27.14 & 21.90 & 467 & 100.00 & 41264 & 592.33 & 28.57 & 23.33 & 468 & 100.00 & 39959 & 765.87 \\
\hline rgn & 62.12 & 0.16 & 142 & 100.00 & 4044 & 0.65 & 74.72 & 0.10 & 123 & 100.00 & 3325 & 0.60 \\
\hline rout & 39.61 & 4.32 & 434 & 100.00 & 115575 & 214.39 & 32.40 & 4.74 & 447 & 100.00 & 349576 & 651.47 \\
\hline vpm1 & 92.99 & 0.05 & 65 & 100.00 & 6 & 0.06 & 92.99 & 0.05 & 79 & 100.00 & 39 & 0.06 \\
\hline vpm2 & 67.45 & 0.61 & 282 & 100.00 & 2804 & 1.59 & 68.45 & 0.58 & 283 & 100.00 & 1503 & 1.28 \\
\hline a1c1s1 & 26.68 & 52.95 & 500 & 65.31 & 1301327 & 7200.00 & 25.68 & 48.29 & 419 & 66.25 & 1070359 & 7200.04 \\
\hline aflow $40 \mathrm{~b}$ & 36.49 & 20.12 & 224 & 100.00 & 1225113 & 5238.47 & 27.88 & 6.92 & 118 & 92.85 & 1468403 & 7200.01 \\
\hline b1c1s1 & 17.62 & 96.32 & 500 & 69.71 & 271930 & 7200.01 & 17.10 & 59.59 & 340 & 69.86 & 435420 & 7200.00 \\
\hline $\mathrm{b} 2 \mathrm{c} 1 \mathrm{~s} 1$ & 18.65 & 103.46 & 500 & 66.72 & 144884 & 7200.00 & 18.51 & 83.38 & 451 & 65.91 & 189048 & 7200.00 \\
\hline bg512142 & 2.55 & 38.92 & 500 & 48.92 & 558244 & 7200.00 & 2.55 & 34.53 & 489 & 46.30 & 624781 & 7200.01 \\
\hline dano3mip & 0.02 & 439.15 & 6 & 1.04 & 2107 & 7200.08 & 0.03 & 135.57 & 7 & 1.09 & 2681 & 7200.14 \\
\hline danoint & 1.46 & 6.34 & 336 & 68.06 & 540115 & 7200.00 & 1.36 & 6.37 & 355 & 67.94 & 582558 & 7200.01 \\
\hline $\operatorname{dg} 012142$ & 0.01 & 84.45 & 499 & 54.55 & 524882 & 7200.01 & 0.01 & 96.07 & 487 & 51.21 & 557727 & 7200.00 \\
\hline $\mathrm{mkc}$ & 51.04 & 20.40 & 256 & 78.68 & 2781088 & 7200.00 & 53.00 & 56.82 & 237 & 73.72 & 2268773 & 7200.00 \\
\hline momentum1 & 63.31 & 373.60 & 266 & 70.68 & 536 & 7200.12 & 61.68 & 479.82 & 243 & 69.03 & 525 & 7200.16 \\
\hline momentum2 & 40.96 & 914.25 & 238 & 68.85 & 483 & 7200.11 & 38.19 & 1193.88 & 196 & 68.78 & 474 & 7200.42 \\
\hline nsrand-ipx & 60.46 & 56.03 & 227 & 89.68 & 1749585 & 7200.00 & 57.78 & 14.89 & 127 & 84.38 & 1570748 & 7200.00 \\
\hline opt1217 & 29.73 & 2.27 & 293 & 30.93 & 8048994 & 7200.09 & 20.13 & 2.08 & 274 & 24.34 & 7342698 & 7200.00 \\
\hline roll3000 & 32.30 & 32.38 & 415 & 77.18 & 879156 & 7200.01 & 46.63 & 34.70 & 413 & 77.58 & 734294 & 7200.01 \\
\hline set $1 \mathrm{ch}$ & 61.32 & 6.32 & 493 & 89.30 & 7397715 & 7200.00 & 56.60 & 5.84 & 500 & 85.02 & 7234043 & 7200.00 \\
\hline swath & 28.44 & 22.31 & 266 & 57.96 & 994262 & 7200.00 & 27.57 & 19.54 & 163 & 48.14 & 959010 & 7200.00 \\
\hline timtab1 & 46.05 & 2.16 & 494 & 91.43 & 16065016 & 7200.00 & 46.25 & 1.92 & 493 & 94.06 & 16386369 & 7200.00 \\
\hline timtab2 & 32.10 & 4.94 & 495 & 61.76 & 9149961 & 7200.00 & 29.94 & 5.27 & 499 & 58.26 & 9108168 & 7200.00 \\
\hline $\operatorname{tr} 12-30$ & 45.85 & 13.88 & 494 & 55.36 & 6131751 & 7200.00 & 44.89 & 15.68 & 499 & 55.03 & 3613179 & 7200.00 \\
\hline
\end{tabular}


A DETAILED TABLES OF RESULTS FROM SECTION 6.3

Table 10: Detailed results for L\&P-5 and R\&S-5 cuts.

\begin{tabular}{|c|c|c|c|c|c|c|c|c|c|c|c|c|}
\hline \multirow[b]{2}{*}{ instance } & \multicolumn{6}{|c|}{ L\&P-5 } & \multicolumn{6}{|c|}{ R\&S-5 } \\
\hline & $\begin{array}{r}\text { root } \\
\text { gap \% }\end{array}$ & $\begin{array}{r}\text { cut } \\
\text { time }\end{array}$ & \#cuts & $\begin{array}{r}\text { final } \\
\text { gap \% }\end{array}$ & \#nodes & $\begin{array}{l}\text { total } \\
\text { time }\end{array}$ & $\begin{array}{r}\text { root } \\
\text { gap \% }\end{array}$ & $\begin{array}{r}\text { cut } \\
\text { time }\end{array}$ & \#cuts & $\begin{array}{r}\text { final } \\
\text { gap \% }\end{array}$ & \#nodes & $\begin{array}{l}\text { total } \\
\text { time }\end{array}$ \\
\hline 10teams & 100.00 & 35.35 & 227 & 100.00 & 161 & 39.85 & 100.00 & 8.46 & 4 & 100.00 & 161 & 13.00 \\
\hline blend2 & 35.58 & 0.48 & 133 & 100.00 & 832 & 0.74 & 27.50 & 0.40 & 130 & 100.00 & 822 & 0.66 \\
\hline dcmulti & 89.82 & 4.12 & 400 & 100.00 & 157 & 4.51 & 87.97 & 3.81 & 404 & 100.00 & 123 & 4.15 \\
\hline dsbmip & 0.00 & 6.78 & 343 & 100.00 & 13 & 6.97 & 0.00 & 9.71 & 369 & 100.00 & 13 & 9.90 \\
\hline egout & 100.00 & 0.01 & 44 & 100.00 & 0 & 0.02 & 100.00 & 0.01 & 20 & 100.00 & 0 & 0.01 \\
\hline fiber & 90.32 & 5.54 & 329 & 100.00 & 547 & 6.10 & 90.43 & 9.66 & 274 & 100.00 & 509 & 10.22 \\
\hline fixnet6 & 61.46 & 1.98 & 171 & 100.00 & 597 & 3.40 & 54.90 & 2.57 & 191 & 100.00 & 842 & 4.61 \\
\hline flugpl & 97.55 & 0.01 & 57 & 100.00 & 16 & 0.02 & 99.93 & 0.02 & 70 & 100.00 & 4 & 0.02 \\
\hline gen & 98.15 & 0.26 & 67 & 100.00 & 0 & 0.27 & 89.88 & 0.39 & 83 & 100.00 & 0 & 0.41 \\
\hline gesa3 & 67.84 & 9.70 & 500 & 100.00 & 138 & 10.04 & 75.64 & 13.63 & 500 & 100.00 & 65 & 13.99 \\
\hline gesa3_o & 90.76 & 9.10 & 390 & 100.00 & 44 & 9.25 & 94.49 & 11.25 & 349 & 100.00 & 29 & 11.37 \\
\hline khb05250 & 98.79 & 0.57 & 90 & 100.00 & 15 & 0.62 & 98.92 & 0.79 & 104 & 100.00 & 15 & 0.86 \\
\hline $\operatorname{misc06}$ & 99.73 & 0.56 & 89 & 100.00 & 7 & 0.62 & 98.51 & 0.84 & 123 & 100.00 & 9 & 0.89 \\
\hline qnet1 & 56.57 & 19.02 & 464 & 100.00 & 504 & 22.18 & 47.22 & 20.17 & 463 & 100.00 & 608 & 26.60 \\
\hline qnet1_o & 72.82 & 10.69 & 414 & 100.00 & 542 & 15.68 & 74.10 & 14.15 & 421 & 100.00 & 337 & 15.64 \\
\hline rentacar & 0.00 & 2.64 & 34 & 100.00 & 30 & 3.07 & 0.00 & 2.08 & 11 & 100.00 & 30 & 2.52 \\
\hline aflow30a & 48.79 & 6.72 & 409 & 100.00 & 21922 & 49.22 & 42.46 & 6.86 & 396 & 100.00 & 28898 & 59.40 \\
\hline arki001 & 61.78 & 16.07 & 482 & 100.00 & 673970 & 1784.63 & 64.31 & 11.53 & 477 & 100.00 & 177593 & 516.17 \\
\hline bell3a & 62.16 & 0.03 & 29 & 100.00 & 33524 & 2.09 & 62.16 & 0.02 & 26 & 100.00 & 24756 & 1.55 \\
\hline gesa2 & 97.15 & 10.66 & 475 & 100.00 & 361 & 11.07 & 97.66 & 14.39 & 489 & 100.00 & 421 & 14.80 \\
\hline gesa2_o & 97.59 & 9.72 & 446 & 100.00 & 288 & 10.00 & 97.76 & 12.74 & 455 & 100.00 & 327 & 13.09 \\
\hline glass4 & 0.00 & 0.29 & 314 & 100.00 & 2265847 & 532.52 & 0.00 & 0.34 & 305 & 100.00 & 2265847 & 517.79 \\
\hline $\operatorname{mas} 74$ & 9.09 & 0.37 & 182 & 100.00 & 3742849 & 435.87 & 8.71 & 0.23 & 128 & 100.00 & 2904274 & 304.35 \\
\hline $\operatorname{mas} 76$ & 9.36 & 0.27 & 143 & 100.00 & 296498 & 26.33 & 8.89 & 0.12 & 91 & 100.00 & 462411 & 39.71 \\
\hline $\operatorname{misc} 07$ & 2.51 & 1.08 & 246 & 100.00 & 38482 & 21.59 & 4.66 & 1.36 & 264 & 100.00 & 41917 & 25.75 \\
\hline $\bmod 011$ & 5.21 & 17.70 & 117 & 100.00 & 10719 & 81.54 & 0.28 & 8.84 & 3 & 100.00 & 19128 & 84.76 \\
\hline modglob & 66.68 & 2.08 & 325 & 100.00 & 50149 & 25.55 & 71.19 & 1.56 & 297 & 100.00 & 16719 & 10.28 \\
\hline noswot & -0.00 & 0.10 & 179 & 100.00 & 694040 & 104.97 & 0.00 & 0.15 & 194 & 100.00 & 694040 & 104.48 \\
\hline pk1 & 0.00 & 0.08 & 150 & 100.00 & 279380 & 35.95 & 0.00 & 0.07 & 149 & 100.00 & 279380 & 36.09 \\
\hline pp08aCUTS & 88.52 & 2.54 & 339 & 100.00 & 2212 & 4.59 & 90.18 & 2.42 & 364 & 100.00 & 2310 & 5.21 \\
\hline pp08a & 96.36 & 1.26 & 329 & 100.00 & 1406 & 2.43 & 96.63 & 1.29 & 346 & 100.00 & 1048 & 2.22 \\
\hline qiu & 27.89 & 23.71 & 467 & 100.00 & 24090 & 499.77 & 28.58 & 23.90 & 468 & 100.00 & 36651 & 661.02 \\
\hline rgn & 64.17 & 0.12 & 136 & 100.00 & 1299 & 0.25 & 73.95 & 0.10 & 122 & 100.00 & 3013 & 0.46 \\
\hline rout & 31.83 & 5.13 & 453 & 100.00 & 182866 & 308.11 & 34.97 & 4.56 & 435 & 100.00 & 124676 & 257.86 \\
\hline vpm1 & 85.63 & 0.05 & 66 & 100.00 & 60 & 0.07 & 92.99 & 0.05 & 79 & 100.00 & 39 & 0.06 \\
\hline vpm2 & 64.99 & 0.77 & 297 & 100.00 & 4726 & 2.41 & 65.84 & 0.64 & 285 & 100.00 & 5073 & 2.42 \\
\hline a1c1s1 & 30.87 & 61.36 & 500 & 68.13 & 1045248 & 7200.00 & 25.76 & 47.33 & 450 & 62.96 & 1090123 & 7200.00 \\
\hline aflow & 35.31 & 19.76 & 217 & 98.35 & 1543564 & 7200.00 & 27.88 & 6.87 & 118 & 93.14 & 1534693 & 7200.00 \\
\hline b1c1s1 & 18.77 & 80.81 & 500 & 70.20 & 387707 & 7200.00 & 14.71 & 67.37 & 404 & 68.85 & 426192 & 7200.01 \\
\hline $\mathrm{b} 2 \mathrm{c} 1 \mathrm{~s} 1$ & 16.79 & 125.31 & 499 & 66.42 & 132228 & 7200.01 & 18.80 & 87.63 & 447 & 64.69 & 160327 & 7200.01 \\
\hline bg512142 & 3.26 & 44.49 & 500 & 49.50 & 564810 & 7200.01 & 2.71 & 36.36 & 489 & 48.39 & 570106 & 7200.00 \\
\hline dano3mip & 0.02 & 386.52 & 6 & 1.05 & 2133 & 7200.10 & 0.03 & 156.65 & 7 & 1.05 & 2266 & 7200.11 \\
\hline danoint & 1.45 & 7.61 & 350 & 70.03 & 582800 & 7200.00 & 1.42 & 6.37 & 354 & 76.44 & 634243 & 7200.00 \\
\hline dg012142 & 0.01 & 85.08 & 500 & 52.44 & 605850 & 7200.00 & 0.01 & 103.72 & 490 & 42.91 & 587047 & 7200.01 \\
\hline $\mathrm{mkc}$ & 45.74 & 37.54 & 256 & 81.17 & 2462049 & 7200.00 & 47.22 & 32.79 & 264 & 78.68 & 2502733 & 7200.00 \\
\hline momentum1 & 52.81 & 390.39 & 237 & 64.72 & 513 & 7200.15 & 61.68 & 540.23 & 243 & 67.49 & 534 & 7200.16 \\
\hline momentum2 & 41.57 & 1208.50 & 104 & 68.81 & 517 & 7200.22 & 32.37 & 1204.54 & 143 & 68.80 & 478 & 7200.38 \\
\hline nsrand-ipx & 65.38 & 58.13 & 241 & 93.91 & 1752996 & 7200.00 & 56.77 & 13.83 & 139 & 82.88 & 1697580 & 7200.00 \\
\hline opt1217 & 20.90 & 2.11 & 292 & 24.54 & 13017661 & 7200.00 & 17.70 & 1.73 & 266 & 22.87 & 16381688 & 7199.99 \\
\hline roll3000 & 37.85 & 35.77 & 435 & 76.57 & 675800 & 7200.01 & 44.86 & 35.66 & 418 & 84.01 & 992031 & 7200.00 \\
\hline set $1 \mathrm{ch}$ & 63.97 & 6.60 & 495 & 91.12 & 7147307 & 7200.03 & 56.92 & 7.06 & 497 & 84.88 & 6892564 & 7200.00 \\
\hline swath & 28.44 & 29.17 & 273 & 57.70 & 963299 & 7200.00 & 27.40 & 19.88 & 154 & 50.90 & 1020300 & 7200.01 \\
\hline timtab1 & 48.98 & 2.48 & 493 & 98.43 & 16991540 & 7200.00 & 48.66 & 2.08 & 498 & 93.76 & 16073424 & 7200.00 \\
\hline timtab2 & 32.96 & 5.51 & 496 & 62.60 & 9715792 & 7200.00 & 31.98 & 5.32 & 497 & 61.11 & 9078882 & 7200.01 \\
\hline $\operatorname{tr} 12-30$ & 46.16 & 14.91 & 492 & 55.54 & 4977278 & 7200.00 & 41.73 & 17.50 & 495 & 52.27 & 4105357 & 7199.99 \\
\hline
\end{tabular}


Table 11: Detailed results for L\&P-6 and R\&S-6 cuts.

\begin{tabular}{|c|c|c|c|c|c|c|c|c|c|c|c|c|}
\hline \multirow[b]{2}{*}{ instance } & \multicolumn{6}{|c|}{ L\&P-6 } & \multicolumn{6}{|c|}{ R\&S-6 } \\
\hline & $\begin{array}{r}\text { root } \\
\text { gap } \%\end{array}$ & $\begin{array}{r}\text { cut } \\
\text { time }\end{array}$ & \#cuts & $\begin{array}{r}\text { final } \\
\text { gap } \%\end{array}$ & \#nodes & $\begin{array}{l}\text { total } \\
\text { time }\end{array}$ & $\begin{array}{r}\text { root } \\
\text { gap \% }\end{array}$ & $\begin{array}{r}\text { cut } \\
\text { time }\end{array}$ & \#cuts & $\begin{array}{r}\text { final } \\
\text { gap \% }\end{array}$ & \#nodes & $\begin{array}{l}\text { total } \\
\text { time }\end{array}$ \\
\hline 10teams & 100.00 & 36.23 & 227 & 100.00 & 161 & 40.78 & 100.00 & 7.34 & 4 & 100.00 & 161 & 11.86 \\
\hline blend2 & 34.71 & 0.56 & 142 & 100.00 & 928 & 0.87 & 30.57 & 0.39 & 121 & 100.00 & 848 & 0.65 \\
\hline dcmulti & 90.70 & 4.04 & 409 & 100.00 & 76 & 4.31 & 87.60 & 3.96 & 368 & 100.00 & 150 & 4.26 \\
\hline dsbmip & 0.00 & 7.21 & 338 & 100.00 & 13 & 7.40 & 0.00 & 9.77 & 384 & 100.00 & 13 & 9.96 \\
\hline egout & 100.00 & 0.02 & 44 & 100.00 & 0 & 0.02 & 100.00 & 0.01 & 20 & 100.00 & 0 & 0.01 \\
\hline fiber & 91.91 & 5.75 & 323 & 100.00 & 564 & 6.29 & 90.33 & 7.68 & 297 & 100.00 & 500 & 8.23 \\
\hline fixnet 6 & 62.21 & 1.98 & 174 & 100.00 & 451 & 2.52 & 56.95 & 2.38 & 184 & 100.00 & 666 & 4.42 \\
\hline flugpl & 97.55 & 0.01 & 57 & 100.00 & 16 & 0.02 & 99.93 & 0.01 & 70 & 100.00 & 4 & 0.02 \\
\hline gen & 98.15 & 0.26 & 67 & 100.00 & 0 & 0.27 & 95.69 & 0.38 & 85 & 100.00 & 0 & 0.39 \\
\hline gesa3 & 74.28 & 9.76 & 485 & 100.00 & 117 & 10.03 & 79.25 & 12.68 & 499 & 100.00 & 103 & 13.00 \\
\hline gesa3_o & 90.83 & 9.40 & 390 & 100.00 & 43 & 9.55 & 94.82 & 10.24 & 353 & 100.00 & 26 & 10.32 \\
\hline khb05250 & 98.79 & 0.58 & 90 & 100.00 & 15 & 0.63 & 98.92 & 0.78 & 104 & 100.00 & 15 & 0.84 \\
\hline misc06 & 99.73 & 0.58 & 89 & 100.00 & 7 & 0.64 & 98.48 & 0.81 & 121 & 100.00 & 10 & 0.87 \\
\hline qnet1 & 54.04 & 18.51 & 464 & 100.00 & 411 & 20.90 & 45.98 & 18.70 & 467 & 100.00 & 339 & 20.90 \\
\hline qnet1_o & 72.25 & 11.57 & 415 & 100.00 & 360 & 13.23 & 74.46 & 13.68 & 418 & 100.00 & 492 & 18.68 \\
\hline rentacar & 0.00 & 2.62 & 34 & 100.00 & 30 & 3.05 & 0.00 & 2.01 & 11 & 100.00 & 30 & 2.46 \\
\hline aflow30a & 45.41 & 7.52 & 401 & 100.00 & 25406 & 64.44 & 44.87 & 7.14 & 392 & 100.00 & 24630 & 52.77 \\
\hline arki001 & 67.86 & 13.63 & 464 & 100.00 & 538882 & 1634.39 & 63.25 & 13.68 & 481 & 100.00 & 184224 & 482.32 \\
\hline bel13a & 62.16 & 0.03 & 29 & 100.00 & 33524 & 2.12 & 62.16 & 0.02 & 26 & 100.00 & 24756 & 1.54 \\
\hline gesa2 & 97.30 & 10.42 & 449 & 100.00 & 264 & 10.77 & 96.92 & 13.26 & 476 & 100.00 & 692 & 13.98 \\
\hline gesa2_o & 91.78 & 10.09 & 451 & 100.00 & 716 & 11.63 & 97.47 & 11.03 & 452 & 100.00 & 636 & 11.87 \\
\hline glass4 & 0.00 & 0.28 & 314 & 100.00 & 2265847 & 529.79 & 0.00 & 0.34 & 305 & 100.00 & 2265847 & 521.99 \\
\hline $\operatorname{mas} 74$ & 9.09 & 0.38 & 182 & 100.00 & 3742849 & 446.98 & 8.81 & 0.20 & 121 & 100.00 & 3763241 & 406.77 \\
\hline $\operatorname{mas} 76$ & 9.32 & 0.28 & 162 & 100.00 & 297407 & 25.27 & 8.89 & 0.12 & 91 & 100.00 & 462411 & 39.81 \\
\hline $\operatorname{misc} 07$ & 2.51 & 1.13 & 246 & 100.00 & 38482 & 22.14 & 2.15 & 1.47 & 284 & 100.00 & 55238 & 37.27 \\
\hline $\bmod 011$ & 5.21 & 16.53 & 117 & 100.00 & 10719 & 80.07 & 0.28 & 9.76 & 3 & 100.00 & 19128 & 89.14 \\
\hline modglob & 59.46 & 2.26 & 342 & 100.00 & 52224 & 25.63 & 73.33 & 1.65 & 304 & 100.00 & 11299 & 6.41 \\
\hline noswot & -0.00 & 0.10 & 179 & 100.00 & 694040 & 104.97 & 0.00 & 0.15 & 194 & 100.00 & 694040 & 104.87 \\
\hline pk1 & 0.00 & 0.07 & 150 & 100.00 & 279380 & 35.69 & 0.00 & 0.07 & 149 & 100.00 & 279380 & 36.06 \\
\hline pp08aCUTS & 88.15 & 2.87 & 354 & 100.00 & 2187 & 5.54 & 91.29 & 2.27 & 361 & 100.00 & 1482 & 4.37 \\
\hline pp08a & 96.28 & 1.36 & 339 & 100.00 & 1284 & 2.69 & 96.31 & 1.22 & 337 & 100.00 & 1705 & 2.94 \\
\hline qiu & 27.79 & 20.48 & 467 & 100.00 & 25465 & 404.77 & 28.67 & 25.66 & 468 & 100.00 & 30836 & 648.53 \\
\hline rgn & 56.68 & 0.15 & 145 & 100.00 & 2893 & 0.54 & 72.84 & 0.10 & 119 & 100.00 & 2900 & 0.43 \\
\hline rout & 29.73 & 5.83 & 463 & 100.00 & 187844 & 372.87 & 33.06 & 4.63 & 445 & 100.00 & 129373 & 241.08 \\
\hline vpm1 & 85.63 & 0.05 & 66 & 100.00 & 60 & 0.07 & 92.99 & 0.05 & 79 & 100.00 & 39 & 0.06 \\
\hline vpm2 & 66.48 & 0.70 & 283 & 100.00 & 4001 & 2.08 & 64.36 & 0.64 & 285 & 100.00 & 3302 & 1.77 \\
\hline a1c1s1 & 26.09 & 63.17 & 499 & 66.63 & 1154669 & 7200.00 & 21.47 & 48.15 & 408 & 60.90 & 1144082 & 7200.01 \\
\hline aflow $40 \mathrm{~b}$ & 35.31 & 19.48 & 217 & 98.68 & 1609454 & 7200.00 & 27.88 & 7.27 & 118 & 93.29 & 1573342 & 7200.00 \\
\hline $\mathrm{b} 1 \mathrm{c} 1 \mathrm{~s} 1$ & 18.41 & 95.71 & 500 & 71.09 & 356632 & 7200.01 & 16.11 & 66.66 & 386 & 69.15 & 383922 & 7200.00 \\
\hline b2c1s 1 & 16.77 & 113.15 & 499 & 65.55 & 126223 & 7200.02 & 12.60 & 74.06 & 377 & 59.55 & 132862 & 7200.02 \\
\hline bg512142 & 3.33 & 43.77 & 500 & 48.35 & 569857 & 7200.01 & 2.68 & 38.90 & 491 & 49.42 & 635674 & 7200.00 \\
\hline dano3mip & 0.02 & 359.22 & 6 & 1.05 & 2126 & 7200.10 & 0.03 & 129.35 & 7 & 1.07 & 2448 & 7200.07 \\
\hline danoint & 1.31 & 7.57 & 353 & 67.71 & 562057 & 7200.01 & 1.52 & 6.68 & 349 & 63.13 & 518736 & 7200.01 \\
\hline dg012142 & 0.01 & 93.88 & 500 & 47.23 & 464062 & 7200.00 & 0.01 & 110.38 & 496 & 49.68 & 574702 & 7200.00 \\
\hline $\mathrm{mkc}$ & 52.95 & 19.28 & 248 & 81.11 & 2422768 & 7200.01 & 54.09 & 29.23 & 243 & 79.44 & 2689472 & 7200.00 \\
\hline momentum1 & 64.51 & 338.10 & 230 & 70.98 & 513 & 7200.30 & 61.68 & 466.82 & 243 & 69.05 & 539 & 7200.21 \\
\hline momentum2 & 29.43 & 1251.96 & 198 & 68.87 & 519 & 7200.17 & 38.86 & 1224.55 & 201 & 68.81 & 501 & 7200.25 \\
\hline nsrand-ipx & 62.71 & 58.81 & 250 & 88.98 & 1398748 & 7200.00 & 57.26 & 14.70 & 117 & 86.60 & 1510243 & 7200.01 \\
\hline opt 1217 & 25.56 & 3.54 & 348 & 28.26 & 4670112 & 7200.01 & 23.24 & 2.12 & 274 & 26.22 & 7265291 & 7200.00 \\
\hline roll3000 & 56.36 & 34.27 & 408 & 72.82 & 678609 & 7200.01 & 44.00 & 38.01 & 429 & 76.80 & 661057 & 7200.02 \\
\hline set $1 \mathrm{ch}$ & 62.63 & 7.17 & 495 & 91.45 & 6952143 & 7200.07 & 59.18 & 6.76 & 494 & 85.17 & 7119449 & 7200.00 \\
\hline swath & 28.43 & 28.81 & 287 & 58.34 & 1047207 & 7200.01 & 27.90 & 17.89 & 132 & 49.60 & 1025380 & 7200.00 \\
\hline timtab1 & 48.74 & 2.28 & 497 & 96.27 & 16722968 & 7200.00 & 45.19 & 2.23 & 495 & 94.21 & 16694199 & 7200.00 \\
\hline timtab2 & 30.36 & 5.57 & 497 & 61.32 & 9836809 & 7200.00 & 34.21 & 5.99 & 498 & 59.25 & 7902097 & 7200.00 \\
\hline $\operatorname{tr} 12-30$ & 46.12 & 16.42 & 493 & 55.74 & 6162441 & 7200.00 & 41.97 & 17.53 & 498 & 52.62 & 4431694 & 7200.00 \\
\hline
\end{tabular}


Table 12: Detailed results for GMI cuts.

\begin{tabular}{|c|c|c|c|c|c|c|}
\hline \multirow[b]{2}{*}{ instance } & \multicolumn{6}{|c|}{ GMI } \\
\hline & $\begin{array}{r}\text { root } \\
\text { gap \% }\end{array}$ & $\begin{array}{r}\text { cut } \\
\text { time }\end{array}$ & \#cuts & $\begin{array}{r}\text { final } \\
\text { gap } \%\end{array}$ & \#nodes & $\begin{array}{l}\text { total } \\
\text { time }\end{array}$ \\
\hline 10teams & 100.00 & 2.51 & 256 & 100.00 & 112 & 5.59 \\
\hline blend2 & 31.83 & 0.04 & 104 & 100.00 & 852 & 0.27 \\
\hline dcmulti & 70.02 & 0.18 & 342 & 100.00 & 253 & 0.42 \\
\hline dsbmip & 0.00 & 1.35 & 342 & 100.00 & 13 & 1.54 \\
\hline egout & 98.97 & 0.01 & 100 & 100.00 & 4 & 0.01 \\
\hline fiber & 89.78 & 0.20 & 239 & 100.00 & 632 & 0.71 \\
\hline fixnet6 & 48.38 & 0.26 & 191 & 100.00 & 690 & 1.12 \\
\hline flugpl & 15.71 & 0.00 & 74 & 100.00 & 223 & 0.01 \\
\hline gen & 21.91 & 0.05 & 106 & 100.00 & 0 & 0.08 \\
\hline gesa3 & 43.45 & 0.20 & 500 & 100.00 & 264 & 0.52 \\
\hline gesa3_o & 60.88 & 0.13 & 162 & 100.00 & 317 & 0.43 \\
\hline khb0 & 94.84 & 0.10 & 107 & 0.00 & 33 & 0.18 \\
\hline $\operatorname{misc06}$ & 72.66 & 0.10 & 92 & 100.00 & 42 & 0.18 \\
\hline qnet1 & 33.24 & 0.52 & 264 & 100.00 & 664 & 2.56 \\
\hline qnet1_o & 56.44 & 0.49 & 270 & 100.00 & 520 & 3.15 \\
\hline rentacar & 0.00 & 0.20 & 16 & 100.00 & 30 & 0.62 \\
\hline aflow30a & 32.50 & 0.59 & 332 & 100.00 & 65531 & 91.48 \\
\hline arkic & 55.68 & 3.88 & 466 & 100.00 & 190622 & 505.08 \\
\hline bel13a & 62.13 & 0.01 & 40 & & 15844 & 1.57 \\
\hline gesa2 & 72.79 & 0.22 & 458 & & 3371 & 4.63 \\
\hline gesa2_o & 81.75 & 0.24 & 406 & & 5497 & 5.60 \\
\hline glass4 & 0.00 & 0.04 & 258 & 100.00 & 2265847 & 518.47 \\
\hline $\operatorname{mas} 74$ & 8.12 & 0.02 & 56 & 100. & 2795555 & 289.31 \\
\hline as 7 & 7.04 & 0.02 & 47 & & 397116 & 31.54 \\
\hline is & 0.72 & 0.14 & 123 & & 7423 & 4.66 \\
\hline $\bmod 0$ & 3.49 & 0.54 & 115 & .00 & 18757 & 88.43 \\
\hline $\mathrm{b}$ & 62.30 & 0.20 & 353 & 10 & 174053 & 102.61 \\
\hline osw & 0.00 & 0.02 & 149 & 100 & 040 & 104.30 \\
\hline pk1 & 0.00 & 0.05 & 150 & 100.00 & 279380 & 36.12 \\
\hline pp08aCUTS & 66.01 & 0.21 & 432 & 100.00 & 23005 & 14.61 \\
\hline pp08a & 85.81 & 0.14 & 423 & 100.00 & 13323 & 7.89 \\
\hline qiu & 15.55 & 2.63 & 468 & 100.00 & 17795 & 187.19 \\
\hline rgn & 42.57 & 0.02 & 145 & & 2632 & 0.36 \\
\hline rout & 12.53 & 0.29 & 333 & 100.00 & 89627 & 87.84 \\
\hline vpm1 & 71.96 & 0.02 & 105 & 100.00 & 8163 & 1.18 \\
\hline vpm2 & 49.27 & 0.04 & 271 & 100.00 & 7642 & 1.88 \\
\hline a1c1s & 22.40 & 1.01 & 500 & 60.32 & 1463987 & 7200.00 \\
\hline afl & .19 & 0.62 & 99 & 92.09 & 792 & 0.00 \\
\hline $\mathrm{b} 1 \mathrm{c} 1$ & 2.52 & 1.96 & 500 & 70.35 & 691 & 0.01 \\
\hline $\mathrm{b} 2 \mathrm{c} 1$ & 8.27 & 3.57 & 500 & 61.88 & 712 & 0.01 \\
\hline bg51 & 1.60 & 1.90 & 500 & 49.50 & 904100 & 0.01 \\
\hline dano3 & 0.02 & 7.19 & 5 & 1.04 & 2381 & 7200.06 \\
\hline danoint & 1.01 & 0.40 & 348 & 82.06 & 860402 & 7200.00 \\
\hline $\operatorname{dg} 012142$ & 0.01 & 2.87 & 500 & 54.55 & 618284 & 7200.00 \\
\hline $\mathrm{mkc}$ & 36.46 & 1.03 & 181 & 81.17 & 2580451 & 7200.00 \\
\hline momentum1 & 64.52 & 13.34 & 246 & 65.89 & 511 & 7200.30 \\
\hline momentur & 39.14 & 57.54 & 161 & 69.01 & 551 & 7200.08 \\
\hline nsra & 45.22 & 2.63 & 117 & 81.77 & 1551147 & 7200.00 \\
\hline opt1 & 50.27 & 0.45 & 218 & 50.27 & 11579593 & 7200.01 \\
\hline roll13000 & & 1.72 & 278 & & 757635 & 7200.00 \\
\hline set $1 \mathrm{ch}$ & 61.11 & 0.20 & 500 & 89.79 & 6850274 & 7200.01 \\
\hline swath & 28.02 & 0.81 & 91 & 41.24 & 915721 & 7200.00 \\
\hline timtab1 & 31.23 & 0.11 & 500 & 89.77 & 16470738 & 7200.00 \\
\hline timtab2 & 26.43 & 0.16 & 500 & 55.80 & 10265277 & 7200.00 \\
\hline $\operatorname{tr} 12-30$ & 52.52 & 0.19 & 500 & 61.45 & 4074819 & 7200.00 \\
\hline
\end{tabular}

